\title{
On War: The Dynamics of Vicious Civilizations
}

\author{
I. Ispolatov ${ }^{1}$, P. L. Krapivsky ${ }^{2}$, and S. Redner ${ }^{1}$ \\ ${ }^{1}$ Center for Polymer Studies and Department of Physics \\ Boston University, Boston, MA 02215 \\ ${ }^{2}$ Courant Institute of Mathematical Sciences \\ New York University, New York, NY 10012-1185
}

\begin{abstract}
The dynamics of "vicious", continuously growing civilizations (domains), which engage in "war" whenever two domains meet, is investigated. In the war event, the smaller domain is annihilated, while the larger domain is reduced in size by a fraction $\epsilon$ of the casualties of the loser. Here $\epsilon$ quantifies the fairness of the war, with $\epsilon=1$ corresponding to a fair war with equal casualties on both side, and $\epsilon=0$ corresponding to a completely unfair war where the winner suffers no casualties. In the heterogeneous version of the model, evolution begins from a specified initial distribution of domains, while in the homogeneous system, there is a continuous and spatially uniform input of point domains, in addition to the growth and warfare. For the heterogeneous case, the rate equations are derived and solved, and comparisons with numerical simulations are made. An exact solution is also derived for the case of equal size domains in one dimension. The heterogeneous system is found to coarsen, with the typical cluster size growing linearly in time $t$ and the number density of domains decreases as $1 / t$. For the homogeneous system, two different long-time behaviors arise as a function of $\epsilon$. When $1 / 2<\epsilon \leq 1$ (relatively fair wars), a steady state arises which is characterized by egalitarian competition between domains of comparable size. In the limiting case of $\epsilon=1$, rate equations which simultaneously account for the distribution of domains and that of the intervening gaps are derived and solved. The steady state is characterized by domains whose age is typically much larger than their size. When $0 \leq \epsilon<1 / 2$ (unfair wars), a few "superpowers" ultimately dominate. Simulations indicate that this coarsening process is characterized by power-law temporal behavior, with non-universal $\epsilon$-dependent exponents. Some of these features are captured by a deterministic self-similar model, for which the characteristic exponents can be computed easily. The transition point $\epsilon=\epsilon_{c}=$ $1 / 2$ is characterized by slower than power-law coarsening.
\end{abstract}

P. A. C. S. Numbers: $01.75+\mathrm{m}, 02.50 .-\mathrm{r}, 89.90+\mathrm{n}$ 


\section{INTRODUCTION}

\section{(a) Background}

Coarsening phenomena underlie a wide variety of physical processes, such as phase ordering and spinodal decomposition [1-3], growth of breath figures [4,5], spin dynamics [6], and foams $[7,8]$. The latter system is especially interesting, as the basic phenomena are readily observable in many everyday situations. The microscopic rules which govern the dynamics of individual bubbles are simply given in terms of the geometry and yet lead to varied and intriguing macroscopic behavior in the long-time limit. In addition to continuous growth and shrinking of individual bubbles, there are discontinuous bubble "popping" events which lead to rearrangement on a larger scale than individual bubble growth. Another attractive feature of such geometric coarsening processes is that they naturally suggest idealizations which may be exactly soluble. One such example, which can be viewed as a limiting case for breath figure growth, is the coarsening of an array of contiguous domains in one dimension [9-17].

The mechanisms that govern domain evolution in these types of coarsening processes appear to have natural counterparts in social phenomena. For example, the competition between cultures has led to a rich historical record [18] in which certain civilizations are dominant for long time periods only to suddenly disappear. Conversely, other civilizations persist for very long times even though they are relatively small. Motivated by these basic historical facts and by a qualitative appreciation for coarsening phenomena, we introduce and investigate a simple, yet relatively general model for war between vicious civilizations. In our model, domains grow continuously and an encounter between two civilizations leads to a war where the smaller combatant is annihilated, and the larger civilization suffers a specified number of casualties. This model exhibits a rich variety of dynamical behaviors, including the features of extinction and persistence of civilizations.

\section{(b) The War Model}

In our model, space is populated by a collection of civilizations, each of which is represented by a spatial domain of a particular size (or population). Each domain grows either continuously or in discrete steps at a constant rate, in which the boundaries move at velocity $V$. Whenever two civilizations of size $i$ and size $j$ meet (with $j>i$ without loss of generality), they engage in a war where the smaller domain is annihilated, while the larger domain suffers $\epsilon i$ casualties, so that its size changes to $j-\epsilon i$ (Fig. 1). We define the casualties to occur at the battlefront so that the frontier of the winner retreats by a distance $\epsilon i$ in one dimension. Here $\epsilon$ measures the "fairness" of the warfare event; $\epsilon=1$ corresponds to a fair war, in which the winner and loser suffer the same number 
of casualties, and $\epsilon=0$ corresponds to a completely unfair war in which the winner emerges unscathed. Subsequently, the survivor civilization continues its growth until the next war. We are interested in determining the long-time dynamical behavior and the spatial distribution of civilizations under these conditions. Although our model is naive and drastic (since the loser is annihilated), the essential features of growth and sudden diminishment by war are incorporated. Thus suitable generalizations of our model may be appropriate for describing quantitative aspects of social history.

There are two general conditions under which our model leads to interesting asymptotic dynamics. In the heterogeneous warfare process, the system begins with a distribution of nascent domains which subsequently undergo growth and warfare. In the homogeneous process, there is temporally constant and spatially uniform input of point domains which subsequently grow and experience wars whenever two domains meet. From a social perspective, this input could be viewed as arising from the remnants of destroyed civilizations.

\section{(c) Overview of Dynamical Properties}

For the heterogeneous warfare process, we have primarily concentrated on the case $\epsilon=1$, although qualitatively similar results are anticipated for any value of $\epsilon$. This system exhibits coarsening, in which the domain size distribution approaches a scaling form in $x / t$, where $x$ is the domain size and $t$ is the time. Consequently the average domain size, $\langle x(t)\rangle$, grows linearly in time. These qualitative results hold both in the mean-field limit and in one dimension. From the rate equations, the scaling function for the domain size distribution is one-half of a sinusoid. In contrast, simulations in one dimension reveal a scaling function for the size distribution which is triangular in shape.

The homogeneous warfare process exhibits a richer phenomenology which is controlled by the fairness parameter $\epsilon$. For $1 / 2<\epsilon \leq 1$, a steady-state arises, which is characterized by egalitarian competition between domains of similar size, while for $\epsilon<1 / 2$, coarsening occurs in which a few superpowers ultimately dominate. The existence of a threshold between these two regimes can be qualitatively justified by considering the outcome of a war between two neighboring domains with different birth times (or equivalently, sizes). For $\epsilon_{c}=1 / 2$, when two neighboring domains meet and engage in war, the frontier of the survivor after the war will retreat to exactly the same position that existed at the birthtime of the smaller civilization (Fig. 2). Thus for $\epsilon \gtrless 1 / 2$, the frontier of the victor after the warfare event should either advance or retreat, respectively, compared to its position at the birth-time of the weaker combatant. This difference suggests the existence of the aforementioned steady state and coarsening regimes which are separated by $\epsilon_{c}=1 / 2$.

An interesting feature of the steady state regime, $\epsilon>\epsilon_{c}$, is that a non-trivial joint distribution of civilization age and size arises. The connection between these two attributes 
is subtle, since a long-lived civilization, whose age is much greater than its size (in appropriately scaled units where $V=1 / 2$ ), can arise by a sequence of fortuitous events. To characterize this age-size distribution, we have formulated a version of the rate equations which simultaneously accounts for the distribution of domains, as well as the distribution of gaps between domains. This approach appears to provide some exact results for the age-size distribution in one dimension, at least in the analytically tractable case of $\epsilon=1$. For the distribution of domain sizes (integrated over all domain ages), $n(x)$, we find $n(x)=e^{-x / x_{0}}$ with $x_{0}=1 / \sqrt{\mu}$, in excellent agreement with simulations. Here $\mu$ is the rate per unit length at which new civilizations are introduced. More interestingly, the age distribution (integrated over all domain sizes), $n(\tau)$, has an exponential tail, $n(\tau) \propto e^{-\tau / \tau_{0}}$ as $\tau \rightarrow \infty$, but with an anomalously large characteristic age $\tau_{0}$ which is much larger than the typical size. Simulations in one dimension give an even larger value for the ratio $\tau_{0} / x_{0}$, a result which can be attributed to the existence of significant anti-correlations in the ages of neighboring domains. For the case of general $\epsilon$ above the threshold, $\epsilon_{c}<\epsilon \leq 1$, our simulations indicate that basic quantities, such as the average domain size, domain age, and coverage, all eventually reach steady-state values. Correspondingly, both the domain size and age distributions decay exponentially in this regime.

In the complementary regime of $\epsilon<\epsilon_{c}$, warfare events sufficiently favor the victor that superpowers ultimately emerge. The existence of these large domains strongly modifies the effect of the continuous input of small domains, so that a steady state is not achieved. Our simulations indicate a continuous coarsening of domains in which basic time-dependent quantities, such as the mean domain size and the number density of domain, exhibit nonuniversal $\epsilon$-dependent power law behavior in time. In particular, for the extreme limit of $\epsilon=0$, where the victor in a war suffers no casualties, the number of domains and the density of empty space decay as $t^{-\gamma}$ in one dimension, with $\gamma \approx 1 / 3$. A deterministic idealization of the war model is introduced which provides a useful description of this coarsening phenomenon. As $\epsilon$ approaches $\epsilon_{c}$ from below, coarsening becomes slower, as evidenced by the $\epsilon$ dependence of the exponents. For $\epsilon \approx \epsilon_{c}$, marginal behavior occurs in which the average domain size grows extremely slowly, while the average age continues to grow as a power law in time.

In Sec. II, we study the heterogeneous war model with fair wars, $\epsilon=1$. Results from a mean-field theory and numerical simulations are presented. We also treat the special case where domains all have the same initial size, so that both domains disappear in a warfare event. This limiting situation turns out to be exactly soluble in one dimension for both the asymptotic value of the coverage, as well as the domain size distribution. We next turn to the homogeneous process in Sec. III. From the rate equations, the equation of the 
steady-state solution for the joint age-size distribution is obtained, again for the particular case of fair war, $\epsilon=1$. The extension of the rate equations for $\epsilon<1$ is also discussed and an argument for the existence of a transition in the kinetic behavior at $\epsilon=\epsilon_{c}$ is presented. We next present simulation results in one dimension, focusing on general properties as a function of the fairness parameter $\epsilon$. Basic features of the $\epsilon>\epsilon_{c}$ steady-state regime and the $\epsilon<\epsilon_{c}$ coarsening regime are outlined. A deterministic self-similar model is then introduced to help understand the coarsening process for $\epsilon=0$. In Sec. IV, we give a brief summary and discuss several extensions of our model. Various calculational details are given in the Appendices.

\section{HETEROGENEOUS WAR}

\section{(a) Mean-Field Theory}

Consider the mean-field limit, in which pairs of domains are randomly picked to undergo warfare. With this interaction rule, the evolution of the domain size distribution is described by the rate equations [19],

$$
\dot{c}_{k}(t)=\sum_{i=1}^{\infty} c_{i}(t) c_{i+k}(t)-c_{k}(t) \sum_{i=1}^{\infty} c_{i}(t)+\lambda\left(c_{k-1}(t)-c_{k}(t)\right) .
$$

Here $c_{k}(t)$ is the concentration of domains of size $k$ at time $t$. The first term accounts for the gain of $k$-domains due to a war between domains of size $i$ and $i+k$. Similarly, the second term accounts for the loss of $k$-domains because of war between a domain of size $k$ and any other domain. The gain and loss of $k$-domains due to constant growth at rate $\lambda$ are described by the last two terms. We have implicitly assumed that the warfare rate is independent of the combatant sizes. Eq. (1) also assumes a minimal size of unity, so that all sizes are integers. The extension to the continuum case is straightforward; and this description will be employed for the homogeneous war model.

For the system described by Eq. (1), the average civilization size grows linearly with time and the size distribution is one-half of a sinusoid. To derive these results, we first identify the appropriate scaling variable. For this purpose, let us temporarily neglect the effect of war. The size distribution for such peacefully flourishing civilizations is found from the rate equation $\dot{c}_{k}=\lambda\left(c_{k-1}-c_{k}\right)$. This is just the Poisson process, with solution, for the "Adam" initial condition of $c_{k}(t=0)=\delta_{k, 1}$,

$$
c_{k}(t)=\frac{(\lambda t)^{k-1}}{(k-1) !} e^{-\lambda t} \sim \frac{1}{\sqrt{2 \pi \lambda t}} \exp \left[-\frac{(k-\lambda t)^{2}}{\lambda t}\right] .
$$

While the exact result is specific for the monodisperse initial condition, the Gaussian approximation provides universal asymptotics for an arbitrary compact initial size distribution. Thus for peaceful civilizations, the size distribution is peaked around $k=\lambda t$, with 
dispersion $\sqrt{\lambda t}$. Clearly, warfare produces sizes with $k<\lambda t$, so that the size distribution for warring civilizations should be non-vanishing for all $k \leq \lambda t$. This suggests that the appropriate scaling variable is $x=k / \lambda t$, with $0 \leq x \leq 1$. The leading region where $k \approx \lambda t$ can be expected to have a fine structure over an extent of the order of $\sqrt{\lambda t}$, as in the special case of peaceful civilizations. In the following, we ignore this detailed structure.

To solve the rate equations, it proves useful to consider first the civilization number density, $N(t)=\sum_{k \geq 1} c_{k}(t)$, which, from Eq. (2), satisfies

$$
\dot{N}(t)=-\frac{1}{2} \sum_{k=1}^{\infty} c_{k}(t)^{2}-\frac{1}{2} N(t)^{2} .
$$

Asymptotically, the first term on the right-hand side is clearly negligible; therefore, as $t \rightarrow \infty, N(t) \sim 2 / t$. We thus expect that scaling form for the civilization size distribution is

$$
c_{k}(t)=\frac{1}{\lambda t^{2}} \mathcal{C}(x), \quad \text { with } \quad x=\frac{k}{\lambda t} .
$$

The time dependent prefactor in Eq. (4) guarantees that $N(t) \propto t^{-1}$. Furthermore, the relation $N(t) \sim 2 / t$ is quantitatively satisfied if $\int_{0}^{1} d x \mathcal{C}(x)=2$.

Substituting the scaling ansatz into the rate equations, one finds that in the continuous limit, the scaling function satisfies an integro-differential equation of an anti-convolution form,

$$
(1-x) \mathcal{C}^{\prime}(x)=\int_{0}^{1-x} d y \mathcal{C}(y) \mathcal{C}(x+y),
$$

where the prime denotes the differentiating with respect to $x$. We are unable to solve this equation by a systematic approach, and therefore resort to trial-and-error. Since $\mathcal{C}^{\prime}>0$, $\mathcal{C}(x)$ is monotonically increasing in $x$. On physical grounds, we expect that $\mathcal{C}(0)=0$, while analysis of Eq. (5) in the vicinity of $x=1$ gives

$$
\mathcal{C}(x)=\mathcal{C}(1)-(1-x)^{2} \frac{\mathcal{C}(1) \mathcal{C}^{\prime}(0)}{4}+\ldots
$$

i. e., $\mathcal{C}^{\prime}(1)=0$ and $\mathcal{C}^{\prime \prime}(1)<0$. Polynomial test functions fail to satisfy Eq. (5). (For a polynomial, say, of degree $g$, the left-hand side of Eq. (5) is a polynomial of degree $g$ while the right-hand side has degree $2 g+1$ ). We find, however, that the simplest appropriate transcendental test function, $\mathcal{C}(x)=\pi \sin \left(\frac{\pi x}{2}\right)$, solves Eq. (5). (The constants in this expression for $\mathcal{C}(x)$ are chosen to satisfy $\mathcal{C}^{\prime}(1)=0$ and the sum rule for $\mathcal{C}(x)$.) Combining Eq. (6) with the scaling form of Eq. (4) gives the basic result,

$$
c_{k}(t) \simeq \begin{cases}\frac{\pi}{\lambda t^{2}} \sin \left(\frac{\pi k}{2 \lambda t}\right), & k \leq \lambda t \\ 0, & k>\lambda t\end{cases}
$$


From this solution, the moments of the size distribution, $M_{n}(t) \equiv \sum_{k \geq 1} k^{n} c_{k}(t)$ are given by

$$
\begin{aligned}
& M_{0}=\frac{2}{t}, \quad M_{1}=\frac{4 \lambda}{\pi}, \quad M_{2}=\frac{8(\pi-2)}{\pi^{2}} \lambda^{2} t, \ldots \\
& M_{n}=\left(\frac{2^{n+1}}{\pi^{n}} \int_{0}^{\pi / 2} d x x^{n} \sin x\right) \lambda^{n} t^{n-1} .
\end{aligned}
$$

Thus, e. $g ., M_{0}(t)$ is the civilization number density, $M_{1}(t)$ is the mass density, or coverage, $M_{1}(t) \equiv M(t)$; etc.. From Eq. (8), any reasonable measure of the typical domain size, e. $g$., $\left[M_{n}(t) / M_{0}(t)\right]^{1 / n}$, increases linearly with time.

\section{(b) Simulation Results in One Dimension}

A straightforward way to simulate our warfare model in one dimension is by molecular dynamics. Within a continuum and deterministic description for domain growth, one identifies the minimum of the conflict times between all pairs of nearest-neighbor domains at any given stage. The system evolves freely until this minimum conflict time, at which point a war occurs, where one domain (the smaller) disappears and its (larger) nearest neighbor shrinks. After this event, all pairwise conflict times are recomputed and the overall update process is repeated. While simple to implement, this molecular dynamics is relatively inefficient, as the computation time is proportional to the square of the number of domains. We therefore employed an alternative algorithm which leads to a savings of almost two orders of magnitude in time for a system with $10^{5}$ initial civilizations, compared to molecular dynamics [20]. In our approach, we first determine the conflict times for all neighboring civilizations and sort them in ascending order. Instead of re-computing conflict times after each war, we continue to use the pre-sorted times for carrying out successive warfare events until a threshold is reached. This threshold is determined by first computing the new nearest-neighbor conflict time that is created as a result of the current war and comparing this new time, as well as the nearest-neighbor conflict times which are "lost" by the current war, with the next conflict time on the pre-sorted list. If any of these putative times are less than this next pre-sorted time, an inconsistency would arise at the next step. It is then necessary to re-compute and re-order all conflict times. This exhaustive molecular dynamics step needs to be performed relatively rarely, leading to considerable saving in simulation time. It is possible, at the expense of algorithmic simplicity, to eliminate the molecular dynamics step entirely by constantly ordering the list of conflict times as conflict times are created and destroyed in each warfare event.

For a polydisperse initial distribution of civilization sizes, our simulations show that the time-dependent size distribution evolves to a nearly universal scaling form in the asymptotic limit. Details of the initial condition are irrelevant as long as they are not singular in 
character. Our numerical results are based on using a Poisson initial distribution for both the sizes of civilizations and the intervening gaps. However, the shape of the size distribution is influenced by details of the warfare event, such as the location of the removed portion of the victorious domain. In our simulations, this removed portion is adjacent to the battlefront (Fig. 1). Other rules are possible and perhaps natural, e. g., one could define a rule in which the center-of-mass of the survivor remains fixed after the warfare event. Because of this detail dependence of the size distribution, its quantitative characterization is of limited value. For the casualty rule adopted here, the distribution has simple triangular shape (Fig. 3).

An interesting feature from the simulations is that the sizes of nearby domains are virtually uncorrelated. That is, the size correlation function, $C_{s}(r) \equiv\left\langle s_{i} s_{i+r}\right\rangle /\left\langle s_{i}\right\rangle^{2}-1 \approx 0$ for $r>1$, where $s_{i}$ is the size of the $i^{\text {th }}$ domain. For $r=1$, the simulations give $C_{s}(1) \approx$ -0.01 which is at least 3 times larger than the correlation function for any other value of $r$. Thus two large civilizations are less likely to coexist peacefully as nearest neighbors; rather, a large domain is slightly more likely to be surrounded by small neighbors and vice versa.

\section{(c) Equal Size Domains}

For heterogeneous war in one dimension, the case of initial equal-size civilizations is unique because both combatants are eliminated in a war and the equal-size distribution is preserved. This case turns out to be exactly soluble by appealing to a connection with domain coarsening processes (see, e. g., Refs. 9-13), and generalizing the approaches in Refs. 14-17. In domain coarsening, which we may view as being "dual" to war for equal size domains (Fig. 4), the system consists of contiguous domains of arbitrary sizes and coarsening occurs by successive elimination of the smallest domain. When the walls associated with this minimal size domain disappear, the other walls remain fixed. This successive domain elimination corresponds exactly to the pairwise annihilation of the two closest domains in the war model, as illustrated in Fig. 4. Note also that since all civilizations have the same size, their growth rate is immaterial, and models with size-dependent

growth may be solved by the same approach as that used for size independent growth by using the domain length $L$ as the time parameter.

Let $n(l, L) d l$ be the number of neighboring civilizations of size $L$ whose centers are separated by a distance which is within $[l, l+d l]$. Using the equivalence to coarsening, we term the interval between the centers of neighboring civilizations a "domain". The total number of such surviving domains is

$$
\mathcal{N}(L)=\int_{L}^{\infty} n(l, L) d l
$$


It proves useful to normalize this quantity, $f(l, L)=n(l, L) / \mathcal{N}(L)$, and then define the (almost) scaling form, $F(x, L)=L f(l, L)$, with $x=l / L$. The absence of correlations [1517 between domains in the dual coarsening process is crucial since it implies that mean-field rate equation for $F(x, L)$ is exact. This rate equation reads [14]

$$
L \frac{\partial}{\partial L} F(x, L)=F(x, L)+x \frac{\partial}{\partial x} F(x, L)+\theta(x-3) G(L) \int_{1}^{x-2} d y F(y, L) F(x-y-1, L),
$$

where $G(L) \equiv F(x=1, L)$. Eq. (10) can be obtained by a straightforward enumeration of the outcomes that arise from the elimination of the smallest domains (see, e. g., the derivation of Eq. (9) in Ref. 17). For example, the last term in the right-hand side of Eq. (10) describes the formation rate of an $x$-domain by elimination of the smallest domain, of scaled length 1 , which is situated between two domains of scaled lengths $y$ and $x-y-1$. The step function $\theta(x-3)$ ensures that the resulting domain will be at least 3 times larger than the minimal domain.

From the solution to Eq. (10) in the long-time limit, or equivalently, $L \rightarrow \infty$, the asymptotic coverage is

$$
M_{\infty}=\frac{1}{2 e^{\gamma}} \cong 0.28073
$$

where $\gamma \cong 0.5772156$ is Euler-Masceroni constant. (This asymptotic solution, first given in Ref. 14, as well as the full time-dependent solution are detailed in Appendix A). Additionally, the number density $N(L)$ of these equal-size civilizations asymptotically is

$$
N(L) \sim M_{\infty} L^{-1} .
$$

These behaviors for the number density and the coverage are qualitatively similar to the corresponding mean-field results. Additionally, from the complete time-dependent solution of the rate equations in Appendix A, one also finds the asymptotic expansion of the coverage,

$$
M(L)=\frac{1}{2 e^{\gamma}}+\frac{A}{L}+\ldots,
$$

i. e., the first dominant correction decays as $L^{-1}$. Here $L$ is a measure of the physical time, and the coefficient $A$ depends on the details of the initial size distribution.

While it would be interesting to investigate the domain evolution in heterogeneous was for general $0 \leq \epsilon<1$, the equal-size property is lost as the process develops, and an exact treatment does not seem possible. However, the extreme case of completely unfair war, $\epsilon=0$, still enjoys the property that an equal-size distribution remains invariant during the evolution if one defines that one of the combatants (picked randomly) is annihilated in a war while the other remains unchanged. Such a model may be solvable by techniques similar to those employed in the above case of equal size domains engaging in fair war. 


\section{HOMOGENEOUS WARFARE}

We now consider the effect of a temporally and spatially homogeneous input of sizeless civilizations on the dynamics. As discussed in the Introduction, two fundamentally different long time behaviors can occur, depending on the value of the fairness parameter $\epsilon$. For $0 \leq \epsilon<1 / 2$, power-law coarsening occurs, leading to the emergence of a few superpowers. Conversely, for $1 / 2<\epsilon \leq 1$, a steady state arises, with egalitarian competition between comparable size domains. For the latter situation, it is plausible that a meanfield approach might be accurate, since the input leads to a well-mixed state. The rate equations of the previous section are not suitable, however, since the restriction to nearestneighbor interactions is not accounted for. Our goal here is to construct rate equations for the driven one-dimensional process which incorporates the obvious restrictions associated with one spatial dimension. We are able to solve for the steady state of these governing equations in the fair war case of $\epsilon=1$.

\section{(a) Rate Equations for Fair Wars}

In one dimension, civilizations are represented by non-overlapping intervals, with the civilization size equal to the interval length. It is now convenient to assume continuous and deterministic civilization growth in which boundaries move with constant velocity $V$. Thus the random birth times and placement of new civilizations (whose initial size may be taken as zero) are the only sources of randomness.

Consider the fair war case of $\epsilon=1$. To write the rate equations, we first introduce the distribution functions, $n(x, t)$ and $m(x, t)$, which are, respectively, the density of civilizations of size $x$ and the density of inter-civilization gaps of size $x$ at time $t$. The number density of civilizations can be written equivalently as

$$
N(t)=\int_{0}^{\infty} d x n(x, t)=\int_{0}^{\infty} d x m(x, t) .
$$

Thus the fraction of covered space is $M(t) \equiv \int_{0}^{\infty} d x x n(x, t)$, while the fraction of empty space is $E(t) \equiv \int_{0}^{\infty} d x x m(x, t)$, with $M(t)+E(t)=1$.

The rate equations for $n(x, t)$ and $m(x, t)$ are

$$
\begin{aligned}
\left(\frac{\partial}{\partial t}+2 V \frac{\partial}{\partial x}\right) n(x, t) & =4 V m_{0}(t)\left[\int_{0}^{\infty} d y \frac{n(y, t)}{N(t)} \frac{n(x+y, t)}{N(t)}-\frac{n(x, t)}{N(t)}\right]+\mu \delta(x) E(t) \\
\left(\frac{\partial}{\partial t}-2 V \frac{\partial}{\partial x}\right) m(x, t) & =2 \mu \int_{x}^{\infty} d y m(y, t)-\mu x m(x, t)-\frac{2 V m_{0}(t) m(x, t)}{N(t)} \\
& +\frac{2 V m_{0}(t)}{N^{3}(t)} \int_{0}^{x} d z m(z, t) n((x-z) / 2, t) \int_{(x-z) / 2}^{\infty} d y n(y, t)
\end{aligned}
$$


In these equations, $m_{0}(t) \equiv m(x=0, t)$ is the density of gaps of size zero and $\mu$ is the birth rate of new domains per unit length. The spatial derivative term in these equations accounts for the continuous growth of civilizations, in Eq. (14a), and the shrinking of gaps, in Eq. (14b). The right-hand sides account for the evolution as a result of interactions (Fig. 5). The first term on the right-hand side of Eq. (14a) gives the production rate for domains of length $x$ as a result of a war between domains of size $y$ and $x+y$. Such an event occurs only when the gap between these two domains vanishes - hence the factor $m_{0}(t)$. In the mean-field approximation, the rate for this process is proportional to the product of $m_{0}(t)$ and the probabilities $n(y, t) / N(t)$ and $n(x+y, t) / N(t)$; the factor $4 V$ accounts for the two possible locations of the combatants, $(y, x+y)$ and $(x+y, y)$, times the rate $2 V$ at which the gap vanishes. The second term on the right-hand side of Eq. (14a) accounts for wars between an $x$-domain and an arbitrary size right- or left-neighbor. The last term gives the rate at which size-less civilizations are created in empty space. Only this last term is exact a priori, because there is no factorization of multi-particle correlation functions into single particle densities.

The terms on the right-hand side of Eq. (14b) are explained similarly. The first two terms arise from the "fragmentation" of an empty interval due to the input of new civilizations. The gain term accounts for the production of an $x$-gap due to the two ways in which a $y$-gap can be broken into a gap size $y-x$ and $x$ by the input. The second term accounts for the loss of $x$-gaps due to their total rate of breakup as a result of the input. These two terms are, again, presumably exact. The last two terms describe how $m(x, t)$ evolves by war. The loss term arises because a war, which is adjacent to an $x$-gap, leads to the removal of the $x$-gap if the adjoining civilization is the loser. There is a cancelation of a factor of 2 , to account for the two possible locations of the warfare event, with a factor of $1 / 2$, to account for the possibility that the loser may not be adjacent to the gap. The total rate for any war, independent of the size of the combatants, is simply $2 V m_{0}(t)$. Finally, the gain term arises from wars between a civilization of size $(x-z) / 2$ and $y>(x-z) / 2$ which is adjacent to a $z$-gap. Since there are $x-z$ casualties in the war, the initial gap of size $z$ grows to size $x$.

\section{(b) Steady State Properties for Fair Wars}

To determine the steady state properties of these equations, it is first helpful to consider the rate equation for the total number density of civilizations,

$$
\frac{d N(t)}{d t}=-2 V m_{0}(t)+\mu E(t)
$$

This equation is exact; it can be derived directly on physical grounds and also follows by integrating Eq. (14(a)) over all $x$. In the steady state, Eq. (15) becomes $2 V m_{0}=\mu E$. 
Substituting this into the steady-state version of Eq. (14(a)) gives

$$
\frac{d}{d x} n(x)=2 m_{0}\left[\int_{0}^{\infty} d y \frac{n(y)}{N} \frac{n(x+y)}{N}--\frac{n(x)}{N}\right]+m_{0} \delta(x) .
$$

We seek an exponential solution to this equation. The presence of the $\delta$-function implies that $n(x=0)=m_{0}$. Thus we hypothesize that $n(x)=m_{0} e^{-a x}$. It is easily verified that this satisfies Eq. (16). Additionally, from $2 V m_{0}=\mu E=\mu \int_{0}^{\infty} x m(x) d x$ and $n(x)=m_{0} e^{-a x}$, the parameter $a$ is determined by $2 V m_{0}=\mu\left(1-\frac{m_{0}}{a^{2}}\right)$. To complete the solution, we now consider the rate equation for $m(x)$. It is again natural to attempt the same exponential form for the gap distribution, $m(x)=m_{0} e^{-a x}$. Substituting this ansatz into the steady version of Eq. (14(b)), consistency is achieved if $\mu=2 V a^{2}$. Combining with the previous relation gives $m_{0}=\mu / 4 V$. Thus we finally arrive at the steady state solution

$$
n(x)=m(x)=\frac{\mu}{4 V} \exp \left(-x \sqrt{\frac{\mu}{2 V}}\right) .
$$

This gives the steady state number density, $N=\sqrt{\mu / 8 V}$ and coverage, $M=E=1 / 2$. Results from our numerical simulations of the homogeneous warfare model in one dimension are indistinguishable from these results, suggesting that this rate equation approach gives the exact the size distribution.

Consider now the civilization age distribution. This turns out to be a considerably more interesting but subtle characteristic of the steady state. Although the age distribution is asymptotically an exponentially decaying function of age, the characteristic age is much larger than the naive expectation of the characteristic size divided by $V=1 / 2$. Thus a typical civilization survives many wars before it is ultimately extinguished. To determine the age distribution, it is helpful to consider the more fundamental steady-state joint agesize distribution, $n(x, \tau)$, defined as the density of civilizations of size $x$ and age $\tau$, (with $x \leq 2 V \tau)$. From this joint distribution, the steady-state size distribution of civilizations of any age is clearly given by

$$
n(x)=\int_{x / 2 V}^{\infty} d \tau n(x, \tau),
$$

while the steady-state age distribution of any size civilizations is given by

$$
n(\tau)=\int_{0}^{2 V \tau} d x n(x, \tau) .
$$

By the nature of the warfare process, the joint age-size distribution consists of two components,

$$
n(x, \tau)=\mathcal{M}(x, \tau)+\mathcal{I}(\tau) \delta(x-2 V \tau)
$$


The first term accounts for "mature" civilizations which have experienced at least one war. The size of such civilizations is strictly less than the maximum possible size, $x_{\max }(\tau)=$ $2 V \tau$, at a given age $\tau$. The second (singular) term accounts for "innocent" civilizations which have not experienced any war during their lifetimes. The $\delta$-function ensures that these innocents are at the maximum size for a given age. These two components of the agesize distribution obey different rate equations. The equation for the density of innocents is readily soluble and this facilitates the full solution.

The rate equation for the density of innocent civilizations is

$$
\frac{d \mathcal{I}}{d \tau}=-4 V \frac{m_{0}}{N} \mathcal{I}(\tau)+\mu E \delta(\tau)
$$

The two terms account for the net change of innocent civilizations by warfare and input, respectively. Here the fraction of empty space in the steady state, $E=1 / 2$, as derived above. Solving Eq. (20a) yields $\mathcal{I}(\tau)=\frac{1}{2} \mu \exp (-\tau \sqrt{8 V \mu}), i$. e., the age distribution of innocent civilizations is purely exponential.

The rate equation for the density of mature civilizations is

$$
\begin{array}{r}
\left(\frac{\partial}{\partial \tau}+2 V \frac{\partial}{\partial x}\right) \mathcal{M}(x, \tau)=4 V m_{0}\left[\int_{0}^{\tau-x / 2 V} d y \frac{n(y)}{N} \frac{\mathcal{M}(x+y, \tau)}{N}-\frac{\mathcal{M}(x, \tau)}{N}\right] \\
+4 V m_{0} \frac{\mathcal{I}(\tau)}{N} \frac{n(2 V \tau-x)}{N}
\end{array}
$$

where $N=\sqrt{\mu / 8 V}$ is the steady state civilization density. The first term on the righthand side accounts for the gain of mature civilizations size $x$ and age $\tau$ due to a war between a mature $(x+y, \tau)$-civilization and one (either mature or innocent) of size $y$ and arbitrary age. Similarly, the second term accounts for the loss of an $(x, \tau)$-civilization due to its undergoing warfare. The last term accounts for the creation of $(x, \tau)$-civilizations due to an innocent civilization of size $x$ and age $\tau=x / 2 \mathrm{~V}$ experiencing its first war with a civilization of size $2 V \tau-x$ and arbitrary age.

The full steady state civilization density $n(x)$ and the density of innocent civilizations $\mathcal{I}(\tau)$ are already known. Therefore, Eq. (20b) is a linear integro-differential equation with non-constant coefficients. It is possible to reduce Eq. (20b) to the Klein-Gordon equation. This reduction, as well as the solution to the resulting boundary value problem, is detailed in Appendix B. From this solution, we find the steady-state age distribution

$$
n(\tau) \propto \tau^{-3 / 2} \exp [-(3-\sqrt{8}) \tau \sqrt{2 V \mu}]
$$

in the large age limit. Therefore, the characteristic age is a factor $(3-\sqrt{8})^{-1} \approx 5.82$ times larger than the characteristic size divided by the rate of growth $2 V$. Thus a typical 
domain survives approximately 6 wars before its ultimate death. Also, it is possible to show that the average cluster age $\langle\tau\rangle=\int_{0}^{\infty} \int_{0}^{\tau} d \tau d x \tau n(x, \tau)=2 / \sqrt{2 V \mu}$. On the other hand, the constant input at unit rate implies that the domain life expectancy equals $1 / \sqrt{2 V \mu}$. This inequality between life expectancy and average age is what may be expected in the harsh environment defined by our war model. Domains are especially vulnerable close to their time of birth, but become progressively more robust as they grow. This behavior is akin to that of sea turtles which are most susceptible to predation immediately after being hatched. However, if the hatching survives its initial trek into the ocean, it has a reasonable chance of living to an old age.

\section{(c) Steady State Properties for Nearly Fair Wars}

In analogy to the case of exactly fair war, we expect that our equation approach should provide an accurate description for steady-state properties in the regime of nearly fair war, $\epsilon \lesssim 1$. The rate equations in this case are straightforward generalizations of Eqs. (14) which, in the steady state, become (taking $V=1 / 2$ )

$$
\begin{gathered}
\frac{d n}{d x}=2 m_{0}\left[\int_{0}^{x /(1-\epsilon)} d y \frac{n(y)}{N} \frac{n(x+\epsilon y)}{N}-\frac{n(x)}{N}\right]+m_{0} \delta(x), \\
\frac{d m}{d x}=\mu x m(x)-2 \mu \int_{x}^{\infty} d y m(y)+m_{0} \frac{m(x)}{N}-\frac{m_{0}}{N^{3}} \int_{0}^{x} d z m(z) n\left(\frac{x-z}{1+\epsilon}\right) \int_{\frac{x-z}{1+\epsilon}}^{\infty} d y n(y) .
\end{gathered}
$$

While we have been unable to solve these equations, it is possible to show that the character of their solution changes as $\epsilon$ decreases from 1 to 0 . This suggests that steady state exists only for a limited range of $\epsilon>\epsilon_{c}$, while a different type of solution exists otherwise. For this purpose, it is sufficient to consider Eq. (22a). Using the transformation

$$
n(x)=m_{0} \mathcal{P}(\xi), \quad \xi=\frac{m_{0}}{N} x,
$$

one can rewrite the rate equation as

$$
\frac{1}{2} \frac{d \mathcal{P}}{d \xi}=\int_{0}^{\xi /(1-\epsilon)} d \eta \mathcal{P}(\eta) \mathcal{P}(\xi+\epsilon \eta)-\mathcal{P}(\xi)
$$

which is to be solved subject to the boundary condition $\mathcal{P}(\xi=0)=1$. For the case of completely unfair war, $\epsilon=0$, we find the explicit solution

$$
\mathcal{P}(\xi)=\frac{1}{(1+\xi)^{2}} .
$$

This solution has a serious flaw in that the first moment of the distribution $\mathcal{P}(\xi)$ is divergent. However, the first moment of the unscaled size distribution, $n(x, t)$, is the 
fraction of covered space and must clearly be finite. Thus a physically acceptable steadystate solution does not exist for $\epsilon=0$. On the other hand, we have previously seen that Eq. (24) does admit a reasonable steady solution, $\mathcal{P}(\xi)=e^{-\xi}$, when $\epsilon=1$. We therefore conclude that the character of the solution to the rate equation changes for some value of $\epsilon$ between 0 and 1. Unfortunately, we are unable to determine the threshold value of $\epsilon$ below which Eq. (24) has no physically acceptable solution.

It is also worth emphasizing that a rate equation description may not even be applicable when $\epsilon<\epsilon_{c}$. When a steady state exists, there is sufficient empty space available in the system for the steady input to act as a relatively effective mixing mechanism. This supports the notion that a mean-field rate equation approach could provide an exact description of some steady-state properties, as discussed in the following section. On the other hand, if the system coarsens, the fraction of empty space vanishes and the input becomes progressively less successful in giving birth to new domains. Under this circumstance, it is not evident that the time-dependent rate equations have the potential to fully capture the time evolution of the system.

\section{(d) Simulation Results in One Dimension}

To test our analytical predictions for $\epsilon=1$ and to map out the dynamical behavior for general $0 \leq \epsilon \leq 1$, we have performed molecular dynamics simulations for one-dimensional systems with between 1000 to 16000 initial domains, with an input rate of size-less civilizations per unit length $\mu=1 / 2$, and the growth velocity $V=1 / 2$. When a steady state arises, any initial condition would be, in principle, suitable. However, to reduce the extent of the early time regime, we empirically found that a good choice for the initial condition is a Poisson distribution for both the domain and inter-domain gap sizes, each with a characteristic length of unity. A summary of our numerical results is given in Tables 1 and 2 .

For $\epsilon=1$, our analytical predictions for the steady-state values of the coverage, concentration, and also the form of the size distribution are confirmed. In particular, for the size distribution, we find $n(x) \cong e^{-x / x_{0}}$, with $x_{0}$ very close to the exact value of $1 / \sqrt{\mu}=\sqrt{2}$ (Fig. 6). Simulations for the domain age distribution also suggest that the asymptotic tail is exponential, namely $n(\tau) \sim e^{-\tau / \tau_{0}}$, but with $\tau_{0}=24 \pm 2$, which is to be compared with the analytical result from the rate equation $\left.\tau_{0}=((3-\sqrt{8}) \sqrt{\mu})\right)^{-1} \approx 8.23$. The source of this discrepancy appears to be the existence of significant correlations in the ages of neighboring civilizations, a feature which would render the rate equations inaccurate. This correlation arises because the domain age remains unaffected by war, so any age correlations which do develop between neighboring domains persist until all of these domains die. On the other hand, the domain size is affected by wars, so that correlations in domain sizes should be 
inhibited by the evolution itself. To check this hypothesis we measured the size and age correlation functions, $C_{s}(r) \equiv\left\langle s_{i} s_{i+r}\right\rangle /\left\langle s_{i}\right\rangle^{2}-1$ and $C_{\tau}(r) \equiv\left\langle\tau_{i} \tau_{i+r}\right\rangle /\left\langle\tau_{i}\right\rangle^{2}-1$, where $s_{i}$ and $\tau_{i}$ refer to the size and age of the $i^{\text {th }}$ domain, respectively. As anticipated, $C_{s}(r)$ is very close to zero for all $r$. However, the age correlation function $C_{\tau}(r)$ is systematically negative for $r \leq 5$ (Fig. 7), which implies that old civilizations are less likely to coexist close to each other. The absence of size correlations and presence of age correlations suggests that the mean-field approach should be quantitatively accurate for the size distribution but not for the age distribution.

Although the geometrical properties of domains are time independent in the steady state, their properties as a function of domain age are not stationary. This age dependence may provide a useful and deeper characterization of the steady state. One such example which appears especially intriguing is the behavior of the average domain size as a function of the corresponding domain age (Fig. 8). This size grows very slowly and ultimately saturates at a finite value as $t \rightarrow \infty$ which is estimated to be approximately 4 . From the solution to the rate equations, we find that the average size has the asymptotic form $\sqrt{\frac{2 V}{\mu}}(2+2 \sqrt{2})-$ const. $/ \tau$. Thus for a domain to be long-lived, it must not be exceptionally large.

For $\epsilon_{c}<\epsilon<1$, our simulations show that a steady state is eventually reached, but that the time needed to attain this steady state grows as $\epsilon$ approaches $\epsilon_{c}$ from above (Fig. 9). Correspondingly, the steady state values of fraction of empty space, $E$, and the concentration of domains, $N$, become smaller for decreasing $\epsilon$. For example, for $\epsilon=1$ and $\mu=1 / 2$, our analytical results give $E=N=1 / 2$, while for $\epsilon=2 / 3$ and $\mu=1 / 2$, simulations give $E \approx 0.35$ and $N \approx 0.25$. Additionally, the corresponding domain size and age distributions for $\epsilon_{c}<\epsilon<1$ appear to have the same functional forms as in the fair-war limit of $\epsilon=1$. In particular, for $\epsilon=2 / 3$ these distributions are $n(x) \sim e^{-x / x_{0}}$, with $x_{0}=5 \pm 0.5$, and $n(\tau) \sim e^{-\tau / \tau_{0}}$, with $\tau_{0}=300 \pm 50$. This increase in $x_{0}$ and $\tau_{0}$ as $\epsilon \rightarrow \epsilon_{c}$ from above has been expected. As $\epsilon$ is decreased, conflicts become less devastating for the survivors, so that they may grow larger and live longer.

When $\epsilon<\epsilon_{c}$, the system never reaches a steady state (Fig. 9), and the evolution of a finite size system ends when a single superpower occupies the entire space. To quantify this coarsening, we consider several basic quantities including the average domain size $\langle x(t)\rangle$, the average maximum domain size $\left\langle x_{\max }(t)\right\rangle$, the number density of domains $N(t)$, the fraction of empty space $E(t)$, the average domain age $\langle\tau(t)\rangle$, and the exponents associated with their asymptotic behavior:

$$
\left\langle x_{\max }(t)\right\rangle \propto t^{\beta(\epsilon)}, \quad N(t) \propto t^{-\gamma(\epsilon)}, \quad E(t) \propto t^{-\gamma(\epsilon)}, \quad\langle\tau(t)\rangle \propto t^{\zeta(\epsilon)} .
$$


As written, these power laws are found to be non-universal, with $\epsilon$-dependent exponents (Table 1). Further, some of these quantities are interrelated. For example, $\langle x(t)\rangle=\int d x x n(x, t) / \int d x n(x, t)=(1-E(t)) / N(t)$. Therefore $\langle x(t)\rangle \propto 1 / N(t) \propto t^{\gamma(\epsilon)}$. Furthermore, the fraction of empty space $E(t)$ and concentration $N(t)$ have the same time dependence. To establish this, it is helpful to introduce the normalized domain size distribution $p(x, t) \equiv n(x, t) / N(t)$. Numerically, we find that this distribution approaches a stationary form, $p(x, t) \rightarrow p(x)$, in the long time limit, and with a universal exponent (Table 2),

$$
p(x) \propto x^{-\delta} \quad \text { when } \quad x \rightarrow \infty .
$$

This stationarity is, in fact, related to the equivalence between $N(t)$ and $E(t)$. Since the concentration of zero size domains is constant and equal to $\mu$ for each inter-domain gap, and since empty gaps account for a fraction $E(t)$ of the system size, $n(x=0, t)=\mu E(t)$. On the other hand, the time dependence in $n(x, t)=p(x) N(t)$ appears only through $N(t)$. We therefore conclude that $N(t) \sim E(t)$. It should be noted, however, that this relation is valid only if $p(x)$ decreases sufficiently fast for large $x$, so that $\int^{t} p(x) d x$ converges as $t \rightarrow \infty$. Our simulations indicate that this is indeed the case, namely, $p(x) \sim x^{-\delta}$ with $\delta \approx 1.7$ (Fig. 10). It is also possible to relate the distribution exponent $\delta$ with dynamic exponents $\beta$ and $\gamma$ by substituting Eq. (26) into the relation $1-E(t)=\int{ }^{x_{\max }} d x x n(x, t)$. Using $x_{\max }(t) \sim t^{\beta}$, the integral is found to behave as $t^{-\gamma+\beta(2-\delta)}$, which leads to the exponent relation

$$
\beta(2-\delta)=\gamma
$$

Our simulations agree with Eq. (27); e. g., for $\epsilon=0$, we find $\beta=1$, in agreement with the obvious intuition that large domains suffer no damage and therefore must grow linearly in time. Correspondingly, we find $\delta \approx 1.7$, and $\gamma \approx 0.3$. As the fairness parameter $\epsilon$ increases from 0 to $\epsilon_{c}$, our simulations indicate that the exponents $\beta$ and $\gamma$ decrease and both appear to go to zero at $\epsilon=\epsilon_{c}$.

The borderline case of $\epsilon=\epsilon_{c}$ can be expected to lead to marginal behavior which is intermediate to the steady state and coarsening regimes. To first establish that $\epsilon_{c}=0.5$, we examined the evolution of a specially prepared system in which a single domain is 500 times larger than all others. The evolution of this defect domain turns out to be both useful and computationally efficient way to ascertain whether the system is in the steadystate or coarsening regimes. In the steady state, the defect domain will eventually shrink to the average size. Thus $\epsilon_{c}$ can be determined as the point where the defect no longer shrinks. This approach gives $0.50 \leq \epsilon_{c}<0.51$ with relatively small computational effort, suggesting that the value of $\epsilon_{c}$ equals $1 / 2$. For $\epsilon=\epsilon_{c}$, we do find that the average domain age $\langle\tau(t)\rangle$ still grows as a power law in time, $\langle\tau(t)\rangle \propto t^{0.61}$, while other basic observables, 
such as the fraction of empty space $E(t)$, the concentration $N(t)$, and the average size $\langle x(t)\rangle$, exhibit extremely slow variations in time (Fig. 11). On a double logarithmic scale, each of these quantities is nearly linear, and a visual fit to the data suggests an exponent that is approximately 0.1 or less. A more careful analysis reveals a weak but systematic curvature in these data, which suggests that the asymptotic behavior will be slower than a power law. However, the time range of the data is insufficient to permit an unambiguous fit to a logarithmic or other slowly varying time dependence.

\section{(e) Deterministic Self-Similar Model}

The existence of power-law domain coarsening for $\epsilon<\epsilon_{c}$ with a simple relation between length and time scales suggests consideration of a deterministic self-similar version of our war model to explain the coarsening exponents. We discuss here one such example which appears to be particularly suitable for describing the coarsening dynamics for $\epsilon=0$. In this deterministic model, the system starts with a regular array of domains with spacing $\Delta x=1$ at $t=0$ (Fig. 12). The domain walls move with velocity $1 / 2$ so that the first set of warfare events takes place at $t=1$. The outcome is defined to be that every second domain is annihilated while the remaining domains continue unscathed. This can be viewed as arising from an infinitesimal difference in the initial domain sizes. Also at regular time intervals $\Delta t=1^{+}$, new domains are seeded at the same integer spatial positions of the initial domains. Only if the seeding occurs in an empty region does the new domain grow. These rules give rise to a pleasing self-similar pattern of domains which resembles a Sierpinski gasket, except for the filling of large empty spaces by the continuous input.

For this system, it is straightforward to compute the properties of domain. These exhibit strong fluctuations, however, because of the determinism of the model. It is therefore convenient to consider quantities which have been averaged over a finite time range, which we choose to be between $2^{n-1}$ and $2^{n}$. For example, by low-order enumeration, it is easy to verify that between $t=0$ and $t=2^{n}$, the total number of domains in existence over a length $2^{n}$, starting from the left edge of figure 12 , is given by the sequence $2,7,22,67, \ldots$ for $n=2,3,4, \ldots$. Solving this sequence, the time integrated density of these domains is asymptotically given by $\frac{5}{6}\left(\frac{2}{3}\right)^{n}$. If we hypothesize that the density $N(t)$ varies as $t^{-\gamma}$, then the corresponding time integrated density is

$$
\left.\int_{0}^{2^{n}} N(t) d t \sim \frac{t^{1-\gamma}}{1-\gamma}\right|_{0} ^{2^{n}} \propto 2^{n(1-\gamma)}
$$

Equating this time integrated density to $(2 / 3)^{n}$ as determined above, we find the exponent $\gamma=2-\ln 3 / \ln 2 \approx 0.415$. In view of the crudeness of this deterministic model, we regard this exponent value as being in good agreement with the corresponding numerical result of $\gamma \approx 0.33$. 


\section{SUMMARY AND DISCUSSION}

We have introduced an idealized warfare model in which domains grow at a constant rate and where a contact between two domains results in a war, with one or both sides suffering casualties. The long time properties of the model are fundamentally governed by a fairness parameter $\epsilon$ which quantifies the outcome of a war. In a war between domains of size $i$ and $j>i$, the smaller domain is annihilated, while the larger domain emerges with a size $j-\epsilon i$. Thus $\epsilon=1$ corresponds to a fair war where the number of casualties in each domain are equal, while $\epsilon=0$ corresponds to a completely unfair war where the winner suffers no casualties. We have examined the long-time kinetics of this model for: (a) the heterogeneous process, in an initial distribution of domains is specified, and after which evolution by domain growth and intermittent warfare ensues, and (b) the homogeneous process, there is a continuous input of infinitesimal domains which then undergo growth and warfare.

For heterogeneous and fair war $(\epsilon=1)$, the system naturally coarsens, with the number of domains decreasing as $1 / t$ and their average size growing as $t$, so that a constant asymptotic coverage arises. The domain size distribution obeys scaling in a manner consistent with these temporal behaviors. While we have not investigated the extension to unfair war in detail, the case $\epsilon=1$ clearly provides a lower bound to the domain size distribution for unfair wars. Thus it is evident that the same quantitative linear in time coarsening will occur for both fair and unfair wars.

In the heterogeneous process, there is a wider range of phenomenology which is fundamentally controlled by the fairness parameter. From simulations and a heuristic argument, there exists a threshold value $\epsilon_{c}=1 / 2$ which separates a steady state regime, for $\epsilon>\epsilon_{c}$, from a regime of continuous coarsening, for $\epsilon<\epsilon_{c}$. In the steady state, the joint agesize distribution of domains provides a comprehensive characterization. Interestingly, the domain lifetime is relatively large, so that a domain typically survives many wars before eventual death. In the coarsening regime, the winner of a war suffers relatively few casualties which promotes the tendency for the oldest clusters in the system to grow without bound. This coarsening evolves according to non-universal $\epsilon$-dependent power laws in time, in which the density of clusters and the fraction of empty space decay as $t^{-\gamma(\epsilon)}$, and the average domain size grows as $t^{\beta(\epsilon)}$. In the completely unfair war case or $\epsilon=0, \beta(\epsilon)=1$, as is intuitively clear, but as $\epsilon \rightarrow \epsilon_{c}$ from below $\beta(\epsilon)$ appears to vanish. At the threshold $\epsilon_{c}$, there is a very slow evolution of the system which has yet to be understood.

The war model also suggests interesting generalizations; a few possibilities and some of their attendant consequences are outlined below.

(i) Size dependent warfare rates: Suppose that the process $(i, i+k) \rightarrow k$ occurs at a rate 
$R(i, i+k)$ which has a power law dependence on the size difference, $i$. e., $R(i, i+k)=k^{\alpha}$. For fair war, where the losses of each combatant are equal, the rate equations for the heterogeneous version of this process are,

$$
\dot{c}_{k}(t)=\sum_{i=1}^{\infty} k^{\alpha} c_{i}(t) c_{i+k}(t)-c_{k}(t) \sum_{i=1}^{\infty}|k-i|^{\alpha} c_{i}(t)+\lambda\left(c_{k-1}(t)-c_{k}(t)\right) .
$$

The constant growth suggests the scaling variable $x=k / \lambda t$, but the time-dependent prefactor in the domain size distribution may be different than $1 / t$ (compare with Eq. (4)). Making the scaling ansatz $c_{k}(t) \propto t^{-a} \mathcal{C}(x)$ and substituting into Eq. (29), self-consistency obtains only when $a=\alpha+2$. Thus the scaling ansatz is

$$
c_{k}(t)=t^{-(\alpha+2)} \mathcal{C}(x), \quad \text { with } \quad x=\frac{k}{\lambda t} .
$$

This scaling form predicts that the size moments behave as power laws in time, $M_{n}(t) \propto$ $t^{n-\alpha-1}$. Hence, the coverage decays with time for positive $\alpha, M_{1}(t) \sim t^{-\alpha}$, indicating that aggressiveness which grows with size disparity leads to extinction (perhaps a lesson for real civilizations).

We may further generalize to a size-dependent power-law growth rate $\lambda=\lambda_{k}=k^{-\beta}$. In the peaceful limit of growth and no war, the size distribution is peaked around $K(t)=$ $[(1+\beta) \lambda t]^{1 /(1+\beta)}$, while warfare produces civilizations in the size range $0<k \leq K(t)$. This suggests the scaling ansatz

$$
c_{k}(t)=t^{-\frac{\alpha+\beta+2}{\beta+1}} \mathcal{C}(x), \quad \text { with } \quad x=\frac{k}{K(t)} \leq 1
$$

which leads to size moments $M_{n}(t) \sim t^{(n-\alpha-\beta-1) /(\beta+1)}$. These results are expected to be valid only for $\beta>-1$. For $\beta=-1$, the typical size grows exponentially, while for $\beta<-1$ the typical size diverges in a finite time, $i$. e., the most aggressive civilization covers the system.

(ii) Bipolar world: Consider two mutually antagonistic species $A$ and $B$ with aggregation occurring when two same-species civilizations (allies) meet, and war occurring when dissimilar species meet. If both species grow at the same constant rate, the mean-field evolution of $A$-civilizations is described by the rate equations (in the heterogeneous case)

$$
\begin{array}{r}
\dot{a}_{k}(t)=\sum_{i=1}^{\infty} b_{i}(t) a_{i+k}(t)-a_{k}(t) \sum_{j=1}^{\infty} b_{j}(t)+\frac{1}{2} \sum_{i=1}^{k-1} a_{i}(t) a_{k-i}(t) \\
-a_{k}(t) \sum_{j=1}^{\infty} a_{j}(t)+\lambda\left(a_{k-1}(t)-a_{k}(t)\right),
\end{array}
$$


and similarly for $B$-civilizations. Here $a_{k}(t)$ and $b_{k}(t)$ are the concentrations of $A$ - and $B$-civilizations of size $k$ at time $t$. The first two terms on the right-hand side account for warfare, the next two terms account for aggregation, and growth is described by the last term.

For this process, it is straightforward to determine that the total number of civilizations $N(t) \simeq 1 / t$. However, the identification of the appropriate variable in a scaling ansatz for the domain size distribution is unclear. In the absence of growth $(\lambda=0)$, a previous study of the resulting aggregation-annihilation process found that the typical size scales as $k \sim \sqrt{t}$ [21]. On the other hand, growth without aggregation and war leads to a size distribution which is peaked around $k \sim \lambda t$, while combined constant growth and aggregation, but without war [22], leads to a typical size which grows as $t \ln t$. A similar ambiguity exists in one dimension since in single-species aggregation and growth, the typical size grows as $e^{t}$ [23]. The homogeneous version of the bipolar world model is also of interest; a preliminary treatment seems to indicate that a steady state does not arise even in the case of fair war.

(iii) War in two dimensions: It is clearly more realistic to consider our war model in two dimensions, where geometrical effects naturally play a more prominent role in defining the outcome of a warfare event. If one posits that war is a localized event at the point of contact between two domains, then the continued action of war will lead to irregularlyshaped domains and possibly to the breakup of countries. These are features which are perhaps best investigated by simulations.

There is a natural simplification which would eliminate the technical difficulties associated with irregularly-shaped domains, however. Namely, start with disk-shaped countries and define that after each war the victor retains a disk shape with its center remaining fixed. A further simplification is to consider the situation where all domains have the same size. In analogy with the corresponding one-dimensional system, analytical progress may be possible. For the equal-size case, intuition suggests that the coverage will approach a constant value $M_{\infty}$ in the long-time limit. Under the further assumption of spatial homogeneity, this would suggest that the number density of civilizations of radius $R, N(R)$, will vary as $\sim M_{\infty} / R^{2}$.

\section{ACKNOWLEDGMENTS}

One of us (S.R.) wishes to thank Glen Swindle for stimulating discussions and the hos-

pitality of the Aspen Center of Physics, where this work was initiated. We also gratefully acknowledge grants from the ARO and NSF for partial support of this research. 


\section{APPENDIX A}

We wish to solve the rate equation

$$
L \frac{\partial}{\partial L} F(x, L)=F(x, L)+x \frac{\partial}{\partial x} F(x, L)+\theta(x-3) G(L) \int_{1}^{x-2} d y F(y, L) F(x-y-1, L),
$$

that describes the dynamics of equal-size growing domains which mutually annihilate when they meet. Here $F(x, L)=L n(l, L) / \mathcal{N}(L)$, where $n(l, L) d l$ is the number of neighboring civilizations whose centers are separated by a distance which is between $[l, l+d l], \mathcal{N}(L)=$ $\int_{L}^{\infty} n(l, L) d l$ is the total number of surviving civilizations, $G(L)=F(x=1, L)$, and $x=l / L$.

To solve Eq. (A1) we apply the Laplace transform,

$$
\Phi(p, L)=\int_{1}^{\infty} d x e^{-p x} F(x, L) .
$$

Note that the relation $\mathcal{N}(L)=\int_{L}^{\infty} n(l, L) d l$ can be rewritten as

$$
\Phi(0, L)=\int_{1}^{\infty} d x F(x, L)=1
$$

Combining Eq. (A2) and Eq. (A1) gives

$$
\left(L \frac{\partial}{\partial L}+p \frac{\partial}{\partial p}\right) \Phi(p, L)=-G(L) e^{-p}\left(1-\Phi^{2}\right) .
$$

Eq. (A4) has been solved previously in the scaling limit of $L \rightarrow \infty$, where it reduces to the ordinary differential equation [14]

$$
p \frac{d \Phi}{d p}=-G_{\infty} e^{-p}\left(1-\Phi^{2}\right),
$$

whose solution is

$$
\Phi(p)=\tanh \left(G_{\infty} \int_{p}^{\infty} \frac{e^{-q}}{q} d q\right)
$$

which contains an as yet undetermined numerical factor $G_{\infty}$. This constant is found from a consideration that also establishes the coverage. Civilizations cover the same space, $x=1$, in units of scaled length, so the coverage is clearly

$$
M(L)=\frac{\int_{1}^{\infty} d x F(x, L)}{\int_{1}^{\infty} d x x F(x, L)} \equiv \frac{1}{\langle x\rangle_{L}} .
$$

Here we use Eq. (A3) and define $\langle x\rangle_{L}$ by $\langle x\rangle_{L}=\int_{1}^{\infty} d x x F(x, L)$. 
In the long-time limit, we use the relation

$$
\int_{p}^{\infty} \frac{e^{-q}}{q} d q=--\ln p-\gamma-\sum_{n=1}^{\infty} \frac{(-p)^{n}}{n \cdot n !}
$$

to expand $\Phi(p)$ in the small $p$ limit as $\Phi(p)=1-2 \exp \left(2 G_{\infty} \gamma\right) p^{2 G_{\infty}}+\ldots$. On the other hand, from the definition of $\Phi(p)$ given in Eq. (A2), we have the expansion, $\Phi(p)=$ $1-p\langle x\rangle_{\infty}+\ldots$. Comparing these two forms gives the constant $G_{\infty}=1 / 2$ and the (scaled) distance between neighboring civilizations, $\langle x\rangle_{\infty}=2 \exp (\gamma)$ [14]. This yields the coverage in the long-time limit $M_{\infty}=1 /\langle x\rangle_{\infty} \cong 0.28073$, i. e., Eq. (11). Having established the asymptotic coverage, the number density $N$ of these equal-size civilizations asymptotically is $N(t) \sim M_{\infty} t^{-1}$ in agreement with Eq. (12).

Now consider the the full time-dependent behavior for which we have to solve the nonlinear partial differential equation Eq. (A4). From the form of the asymptotic solution of the time independent equation, it is natural to attempt the ansatz

$$
\Phi(p, L)=\tanh [\Psi(p, L)]
$$

which allows us to eliminate the nonlinear factor $\left(1-\Phi^{2}\right)$. Substituting Eq. (A9) into Eq. (A4) gives

$$
\left(L \frac{\partial}{\partial L}+p \frac{\partial}{\partial p}\right) \Psi(p, L)=-G(L) e^{-p} .
$$

Transforming from the variables $(p, L)$ to $u=\sqrt{p L}$ and $v=\sqrt{p / L}$ simplifies Eq. (A10) to

$$
u \frac{\partial}{\partial u} \Psi(u, v)=-G\left(\frac{u}{v}\right) e^{-u v} .
$$

The solution to Eq. (A11) is now straightforward,

$$
\Psi(u, v)=\int_{u}^{\infty} \frac{d \xi}{\xi} G\left(\frac{\xi}{v}\right) e^{-\xi v}+\chi(v),
$$

up to an arbitrary function $\chi(v)$. To determine $\chi(v)$, note that the definition of $\Phi(p, L)$, (Eq. (A2)), implies $\Phi(p, L) \simeq p^{-1} e^{-p} G(L)$ in the large- $p$ limit. Since $G(L)$ varies over a limited range (as it is clear, e. $g$., from relation $G(\infty)=1 / 2$ ), we conclude that $\Phi(p, L) \rightarrow 0$ and hence, $\Psi(p, L) \rightarrow 0$ as $p \rightarrow \infty$. Choose now $p \sim L \rightarrow \infty$; in the $(u, v)$ variables, this corresponds to $u \rightarrow \infty$ and $v$ finite. Thus the integral in Eq. (A12) disappears in this limit and we find $\Psi(\infty, v)=\chi(v)=0$ implying that $\chi(v)$ is trivial.

Returning now to original variables and replacing $\xi$ by $\eta$ defined via $\xi=\eta u$, we rewrite Eq. (A12) as

$$
\Psi(p, L)=\int_{1}^{\infty} \frac{d \eta}{\eta} G(L \eta) e^{-p \eta}
$$


We have thus solved Eq. (A2), up to an as yet unknown function $G(L)$. This function can be found, in principle, from the initial conditions. Technically, it is convenient to assume that there is a finite small-size cutoff $L_{\min }$ in the initial distribution which we set to be $L_{\text {min }}=1$ without loss of generality. As an example initial distribution, consider a shifted Poisson

$$
F(x, 1)= \begin{cases}e^{-(x-1)}, & x \geq 1 \\ 0, & x<1\end{cases}
$$

In this case $\Phi(p, 1)=(1+p)^{-1} e^{-p}$; therefore, $G(L)$ is determined from the following equation:

$$
\int_{1}^{\infty} \frac{d \eta}{\eta} G(\eta) e^{-p \eta}=\operatorname{Arctanh}\left[(1+p)^{-1} e^{-p}\right]
$$

Although it is impossible to find explicit expression for $G(L)$ in terms of elementary functions, one can readily compute asymptotics behaviors, e. $g ., M_{\infty}-M(L) \propto L^{-1}$.

Thus the model of equal-size warring civilizations is exactly solvable in one dimension. While asymptotic characteristics have been computed by exploiting previously known results, the complete solution for arbitrary time is new. However, several related and interesting properties have not yet been computed. One such quantity is the density of feral space, $i$. e., the fraction of space that has been untouched by any civilization.

\section{APPENDIX B}

We outline here a solution to Eqs. (20) for the joint age-size domain distribution. For this purpose, it proves convenient to rescale length and time by $x \rightarrow x \sqrt{2 V / \mu}$ and $\tau \rightarrow \tau / \sqrt{2 V \mu}$. In these rescaled units, the previous results for the size distribution become

$$
n(x)=\frac{e^{-x}}{2}, \quad m_{0}=N=\frac{1}{2} .
$$

Then Eq. (20a) simplifies to $\frac{d \mathcal{I}}{d \tau}=-2 \mathcal{I}(\tau)+\frac{1}{2} \delta(\tau)$, whose solution is

$$
\mathcal{I}(\tau)=\frac{e^{-2 \tau}}{2}
$$

This shows that in the steady state regime the number density of innocent civilizations, $I=\int_{0}^{\infty} d \tau \mathcal{I}(\tau)=\frac{1}{4}$, is one-half of the total number density, $I=N / 2$. Using Eqs. (B1)(B2), we now reduce the rate equation (20b) to

$$
\left(\frac{\partial}{\partial \tau}+\frac{\partial}{\partial x}\right) \mathcal{M}(x, \tau)=2\left[\int_{0}^{\tau-x} d y e^{-y} \mathcal{M}(x+y, \tau)-\mathcal{M}(x, \tau)\right]+e^{x-3 \tau} .
$$

Introducing $g(x, \tau)=e^{3 \tau-x} \mathcal{M}(x, \tau)$, reduces Eq. (B3) to

$$
\left(\frac{\partial}{\partial \tau}+\frac{\partial}{\partial x}\right) g(x, \tau)=2 \int_{x}^{\tau} d y g(y, \tau)+1
$$


Defining now $f(x, \tau) \equiv 2 \int_{x}^{\tau} d y g(y, \tau)+1$, Eq. (B4) becomes

$$
\left(\frac{\partial^{2}}{\partial x \partial \tau}+\frac{\partial^{2}}{\partial x^{2}}\right) f=-2 f(x, \tau) .
$$

One boundary condition, $f(x=\tau, \tau)=1$, follows directly from the definition of $f$. To obtain a second condition, we compare total number of zero-size civilizations, $n(x=0)$, with the number of innocent zero-size civilizations, $\mathcal{I}(x=0)$. Both quantities are equal to $1 / 2$ which means that $\int_{0}^{\infty} \mathcal{M}(x=0, \tau) d \tau=0$. Since $\mathcal{M}(x, \tau)$ is nonnegative for all $\tau \geq 0$, we conclude that $\mathcal{M}(x=0, \tau)=0$, which leads to the boundary condition $\left.\frac{\partial f}{\partial x}\right|_{x=0}=0$.

Eq. (B5) simplifies further after the change of variables, $(x, \tau) \rightarrow(\alpha, \beta)=(\tau, \tau-x)$ :

$$
\frac{\partial^{2} f}{\partial \alpha \partial \beta}=2 f(\alpha, \beta) .
$$

This Klein-Gordon equation is to be solved in the region $\alpha \geq \beta \geq 0$, with the boundary conditions

$$
\left.f\right|_{\beta=0}=1, \quad \text { and }\left.\quad \frac{\partial f}{\partial \beta}\right|_{\alpha=\beta}=0 .
$$

The symmetry of the governing equation under the exchange of the variables, $\alpha \leftrightarrow \beta$, suggests seeking a symmetric solution which depends on a single variable $\alpha \beta$. Further analysis indicates that the variable $z=\sqrt{8 \alpha \beta}$ is especially convenient. Substituting $f=$ $f(z)$ reduces the Klein-Gordon equation to the modified Bessel equation,

$$
f^{\prime \prime}+\frac{1}{z} f^{\prime}-f=0
$$

where the prime denotes differentiation with respect to $z$. A potential solution is $f=$ $I_{0}(z)$. This satisfies the boundary condition Eq. (B6); however, the boundary condition of Eq. (B7) is not satisfied. To remove this drawback, we make use of the linearity of the governing equation and seek a solution of the form

$$
f(\alpha, \beta)=I_{0}(z)+\beta^{n} g(z)
$$

The first of the boundary conditions in Eq. (B6) is manifestly satisfied (when the index $n$ is positive). Substituting Eq. (B9) into Eq. (B6) we get

$$
g^{\prime \prime}+\frac{2 n+1}{z} g^{\prime}-g=0
$$

Eq. (B10) is readily solved to find $g(z)=A z^{-n} I_{n}(z)$, where $I_{n}(z)$ is the modified Bessel function of order $n$. To determine the index $n$ and the amplitude $A$ we substitute Eq. (B9), 
with $g(z)=A z^{-n} I_{n}(z)$, into the second boundary condition in Eq. (B7). This yields the relation

$$
\sqrt{2} I_{1}(z)+2^{\frac{1-3 n}{2}} A I_{n-1}(z)=0 .
$$

In deriving Eq. (B11) we used the identities [24]

$$
\begin{aligned}
& I_{n-1}(z)-I_{n+1}(z)=\frac{2 n}{z} I_{n}(z), \\
& I_{n-1}(z)+I_{n+1}(z)=2 I_{n}^{\prime}(z),
\end{aligned}
$$

and the equality $z=\beta \sqrt{8}$ on the diagonal $\alpha=\beta$. Eq. (B11) shows that $n=2$ and $A=-8$. Thus we determine the desired solution to the Klein-Gordon equation with mixed boundary conditions

$$
f(\alpha, \beta)=I_{0}(z)-\frac{\beta}{\alpha} I_{2}(z) .
$$

Returning to the original variables $(x, \tau)$, and the original joint age-size distribution function $n(x, \tau)$, we get after straightforward computations

$$
n(x, \tau)=\mathcal{I}(x, \tau)+\mathcal{M}(x, \tau)=2 x e^{x-3 \tau} \frac{I_{1}(z)}{z}+\frac{e^{-2 x}}{2} \delta(x-\tau), \quad z \equiv \sqrt{8(\tau-x) \tau} .
$$

The sum rule Eq. (18a) provides a useful self-consistency check. Substituting Eq. (B14) into $n(x)=\int_{x}^{\infty} d \tau n(x, \tau)$ gives

$$
\frac{e^{-x}}{2}-\frac{e^{-2 x}}{2}=\int_{x}^{\infty} d \tau \frac{x e^{x-3 \tau}}{\sqrt{2(\tau-x) \tau}} I_{1}(\sqrt{8(\tau-x) \tau}) .
$$

This identity is indeed satisfied [25]. Another quantity which can be calculated exactly is average age, $\langle\tau\rangle \equiv \int_{0}^{\infty} n(\tau) \tau d \tau$, where $n(\tau)=\int_{0}^{\tau} d x n(x, \tau)$ (Eq. (18b)). Changing the order of integration and using [25], we obtain that $\langle\tau\rangle=2$.

Let us finally consider the age distribution of mature civilizations $\mathcal{M}(\tau)$. It is given by $\mathcal{M}(\tau)=\int_{0}^{\tau} d x \mathcal{M}(x, \tau)$. In the large-age limit we use the asymptotic relation [24] $I_{1}(z) \simeq \frac{e^{z}}{\sqrt{2 \pi z}}$ to estimate the integral. Thus we arrive at

$$
\mathcal{M}(\tau) \simeq \frac{B}{\tau^{3 / 2}} e^{-(3-\sqrt{8}) \tau}, \quad B=\frac{(\sqrt{2}+1)^{2}}{\pi^{1 / 2} \cdot 2^{7 / 4}} \cong 0.977629
$$

Since the age distribution of innocent civilizations decays as $e^{-2 \tau}$, Eq. (B16) indicates that old civilizations are mostly mature. Another interesting computation is the average domain size as a function of age,

$$
\langle x(\tau)\rangle=\frac{\int_{0}^{\tau} d x x n(x, \tau)}{\int_{0}^{\tau} d x n(x, \tau)} .
$$

Using the asymptotic behaviors outlined above, we find

$$
\langle x(\tau)\rangle \sim(2+2 \sqrt{2})\left(1-\frac{\text { const. }}{\tau}\right) .
$$


Table 1

\begin{tabular}{|c|c|c|c|}
\hline$\epsilon$ & $\beta$ & $\gamma$ & $\zeta$ \\
\hline 0.45 & 0.46 & 0.15 & 0.61 \\
\hline 0.4 & 0.55 & 0.18 & 0.59 \\
\hline 0.2 & 0.77 & 0.25 & 0.53 \\
\hline 0 & 1 & 0.33 & 0.35 \\
\hline
\end{tabular}

Table 1. The estimated values of the exponents that characterize the time dependent properties of domains in the coarsening regime, $\epsilon<1 / 2$. The estimated error in these numbers is $5 \%$ or less.

Table 2

\begin{tabular}{|c|c|c|}
\hline$\epsilon$ & $p(x)$ & $p(\tau)$ \\
\hline$>0.5$ & exponential & exponential \\
\hline 0.5 & 1.75 & 1.47 \\
\hline 0.45 & 1.79 & 1.49 \\
\hline 0.4 & 1.72 & 1.51 \\
\hline 0.2 & 1.66 & 1.63 \\
\hline 0 & 1.67 & 1.67 \\
\hline
\end{tabular}

Table 2. The characteristic exponents of the age and size distributions. The estimated error in these numbers is $10 \%$ or less. 


\section{REFERENCES}

1. J. D. Gunton, M. San Miguel, and P. S. Sahni, in Phase Transitions and Critical Phenomena, eds. C. Domb and J. L. Lebowitz (Academic Press, London, 1983), vol. 8.

2. J. S. Langer, in Solids Far From Equilibrium, ed. C. Godrèche (Cambridge University Press, Cambridge, 1992).

3. A comprehensive recent review of the coarsening dynamics is given by A. J. Bray, Adv. Phys. 43, 357 (1994).

4. D. A. Beysens, and C. M. Knobler, Phys. Rev. Lett. 57, 1433 (1986); J. L. Viovy, D. A. Beysens, and C. M. Knobler, Phys. Rev. A 37, 4965 (1988); D. Fritter, C. M. Knobler, and D. A. Beysens, Phys. Rev. A 43, 2858 (1991).

5. P. Meakin, Rep. Prog. Phys. 55, 157 (1992).

6. K. Kawasaki, in Phase Transitions and Critical Phenomena, eds. C. Domb and M. S. Green (Academic Press, London, 1972), vol. 2.

7. D. Weaire and N. Rivier, Contemp. Phys. 25, 59 (1984); J. A. Glazier and J. Stavans, Phys. Rev. A 40, 7398 (1989).

8. J. Stavans, Rep. Prog. Phys. 56, 733 (1993).

9. B. Derrida, C. Godreche, and I. Yekutieli, Phys. Rev. A 44, 6241 (1991).

10. T. Nagai and K. Kawasaki, Physica A 120, 587 (1983); K. Kawasaki and T. Nagai, Physica A 121, 175 (1983).

11. J. Zhuo, G. Murthy, and S. Redner, J. Phys. A 25, 5889 (1992).

12. J. Carr and R. Pego, Proc. Roy. Soc. London Ser. A 436, 569 (1992).

13. S. N. Majumdar and D. A. Huse, Phys. Rev. E 52, 270 (1995).

14. T. Nagai and K. Kawasaki, Physica A 134, 483 (1986); K. Kawasaki, A. Ogawa, and T. Nagai, Physica B 149, 97 (1988).

15. A. D. Rutenberg and A. J. Bray, Phys. Rev. E 50, 1900 (1994).

16. A. J. Bray, B. Derrida, and C. Godreche, Europhys. Lett. 27, 175 (1994).

17. A. J. Bray and B. Derrida, Phys. Rev. E 51, 1633 (1995).

18. See e. g., J. Keegan, A History of Warfare, (Alfred A. Knopf, New York, 1993); L. N. Gumilev, Searches for an Imaginary Kingdom: The Legend of the Kingdom of Prester John, (Cambridge University Press, New York, 1987). 
19. The rate equations and their solution for this competitive process in the absence cluster growth was given in S. Redner, D. ben-Avraham, and B. Kahng, J. Phys. A 20, 1231 (1987).

20. A similar algorithm was developed for the simulation of ballistic annihilation processes in P. L. Krapivsky, S. Redner, and F. Leyvraz, Phys. Rev. E 51, 3977 (1995).

21. P. L. Krapivsky, Physica A 198, 135 (1993); E. Ben-Naim and P. L. Krapivsky, Phys. Rev. E 52, 6066 (1995).

22. P. L. Krapivsky and S. Redner, unpublished.

23. K. Sekimoto, Int. J. Mod. Phys. B 5, 1843 (1991).

24. C. M. Bender and S. A. Orszag, Advanced Mathematical Methods for Scientists and Engineers (McGraw-Hill, New York, 1978).

25. A. P. Prudnikov, Yu. A. Brychkov, O. I. Marichev, Integrals and Series (Gordon and Breach Science Publishers, New York, 1986), v.2, p.309. 


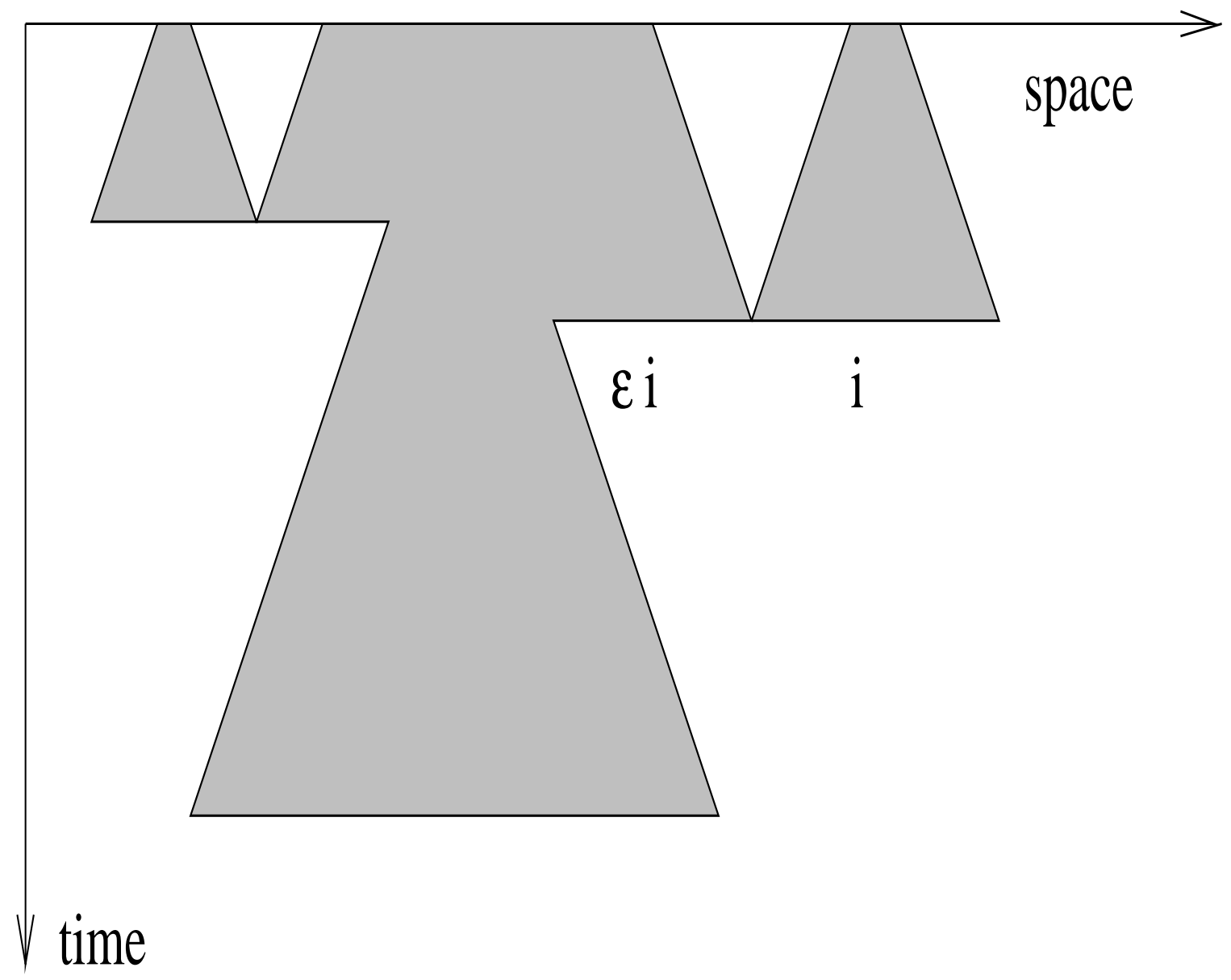

Figure 1. Space-time dynamics of vicious civilizations in one dimension. Shown is the heterogeneous version of the war model where the system evolves from a fixed initial state. When two civilizations of size $i$ and $j>i$ meet, the result is a diminished civilization of size $j-\epsilon i$. By definition, casualties occur at the point of contact, so that the side of the large civilization on the battle front retreats by $\epsilon i$ while the other side is not affected. 


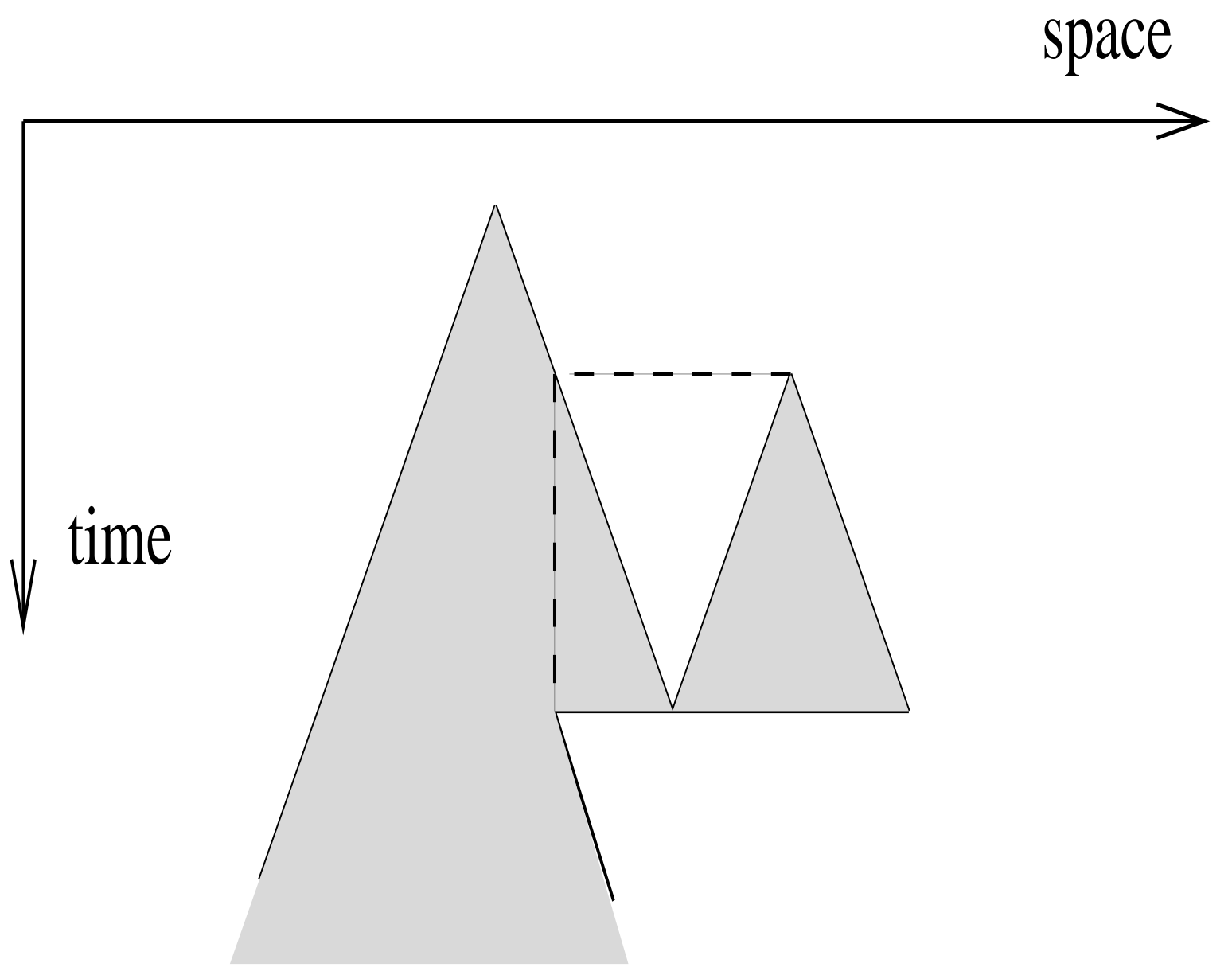

Figure 2. Space-time evolution of two domains for the special case of $\epsilon=1 / 2$. The position of the frontier of the winner immediately after the war and at the birth-time of the smaller combatant is the same. 


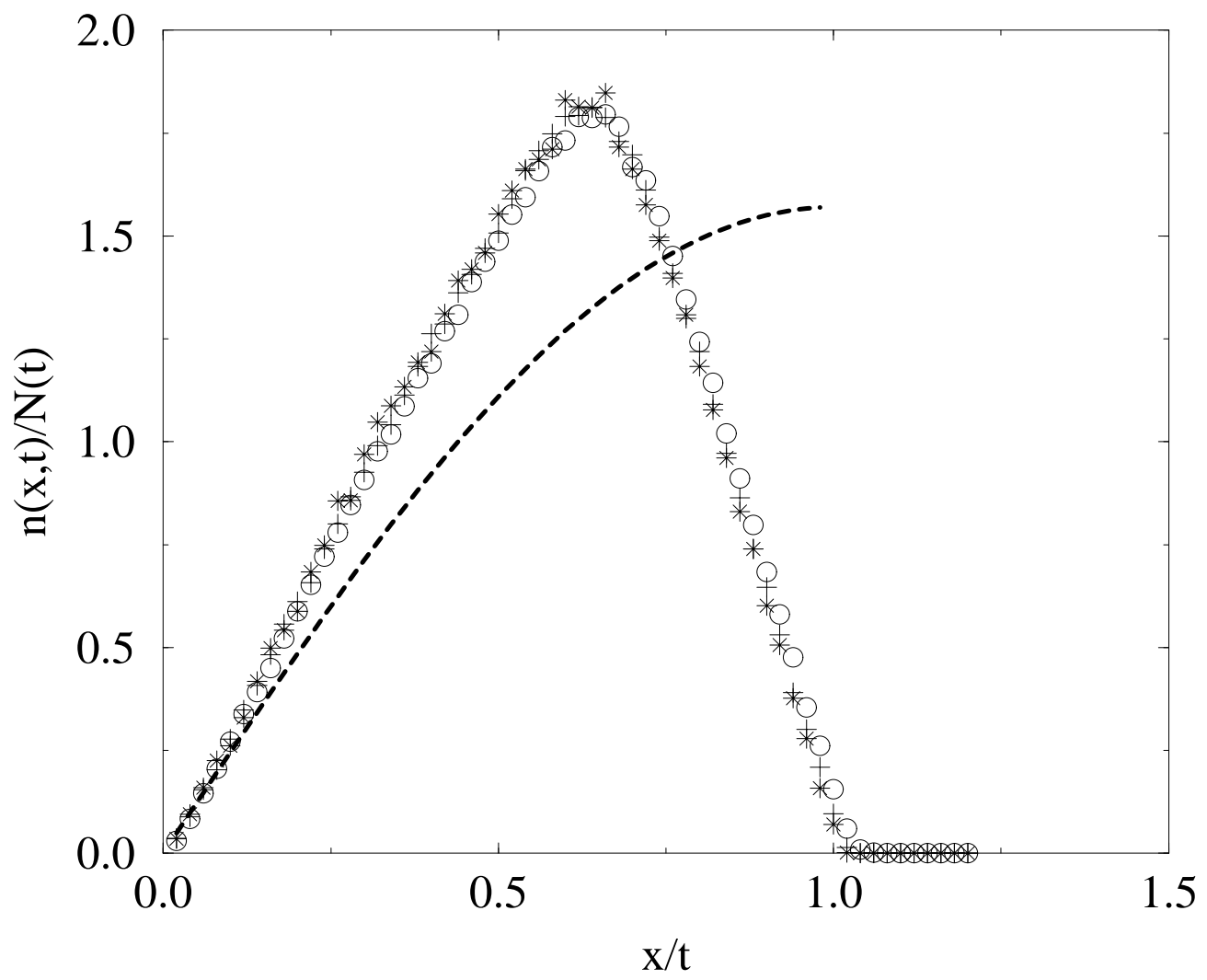

Figure 3. Scaled civilization size distribution, $n(x, t) / N(t)$, from numerical simulations of 500 configurations of heterogeneous war with $10^{5}$ initial domains in one dimension. The data are for $t \approx 1.5^{10} \approx 57.7(\circ), t \approx 1.5^{12} \approx 129.7(+)$, and $t \approx 1.5^{14} \approx 291.9(*)$. Also shown is the corresponding mean-field result $\frac{\pi}{2} \sin \frac{\pi x}{2 t}$ (dashed). 


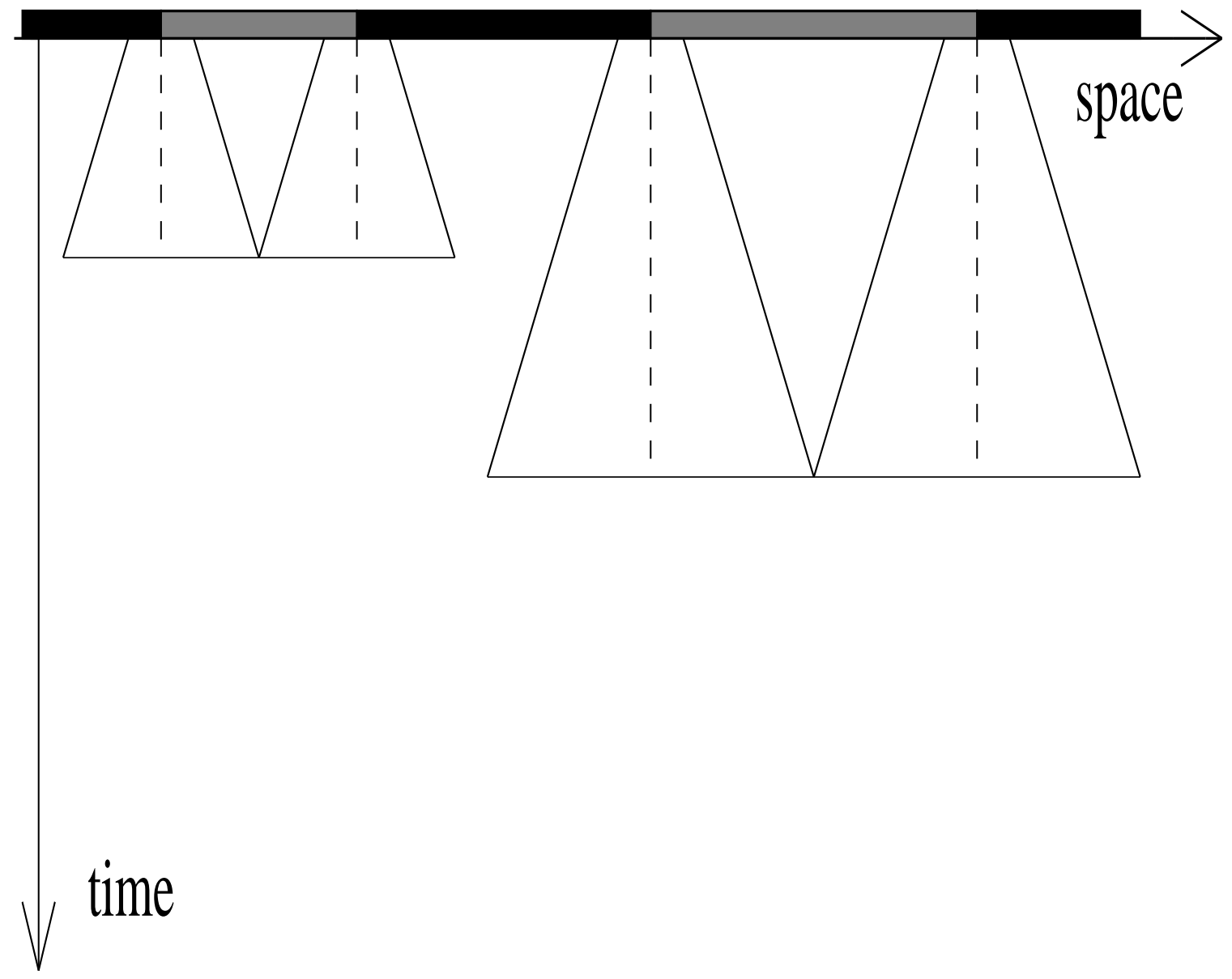

Figure 4. Schematic illustration of the equivalence between the space-time evolution in the onedimensional war model with equal size domains, and the "dual" problem of coarsening of domains through the successive elimination of the smallest domain. In this equivalence, the centers of the domains in the war model are identified with the positions of domain walls in the coarsening process (dashed lines). The two domains which are eliminated in the dual problem are indicated by the dark shading. 


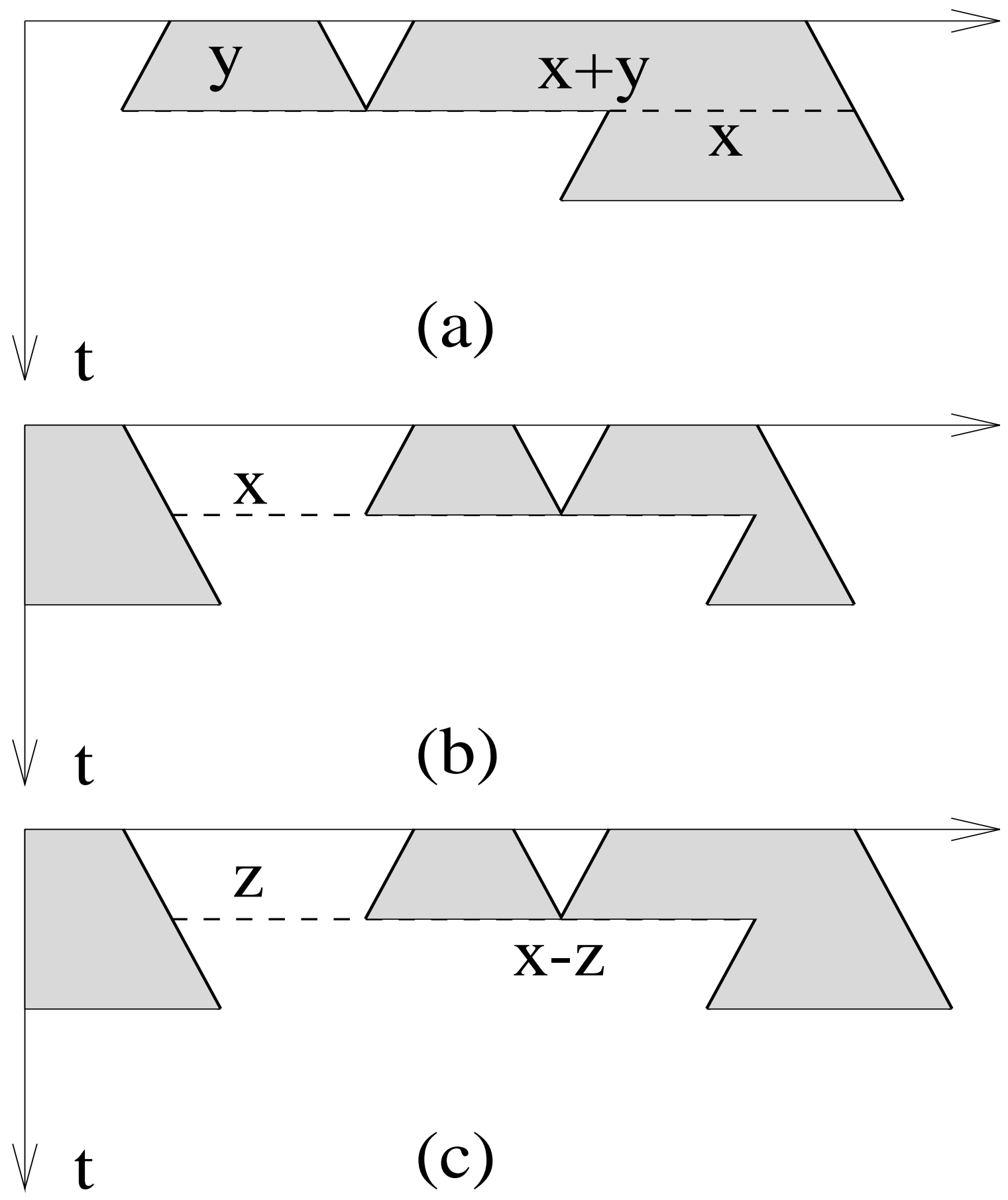

Figure 5. Correspondence between various interaction events and the terms in the rate equation, Eqs. (14). (a) Illustration of the first term on the right-hand side of Eq. (14a), (b) the third and (c) the fourth terms on the right-hand side of Eq. (14b). 


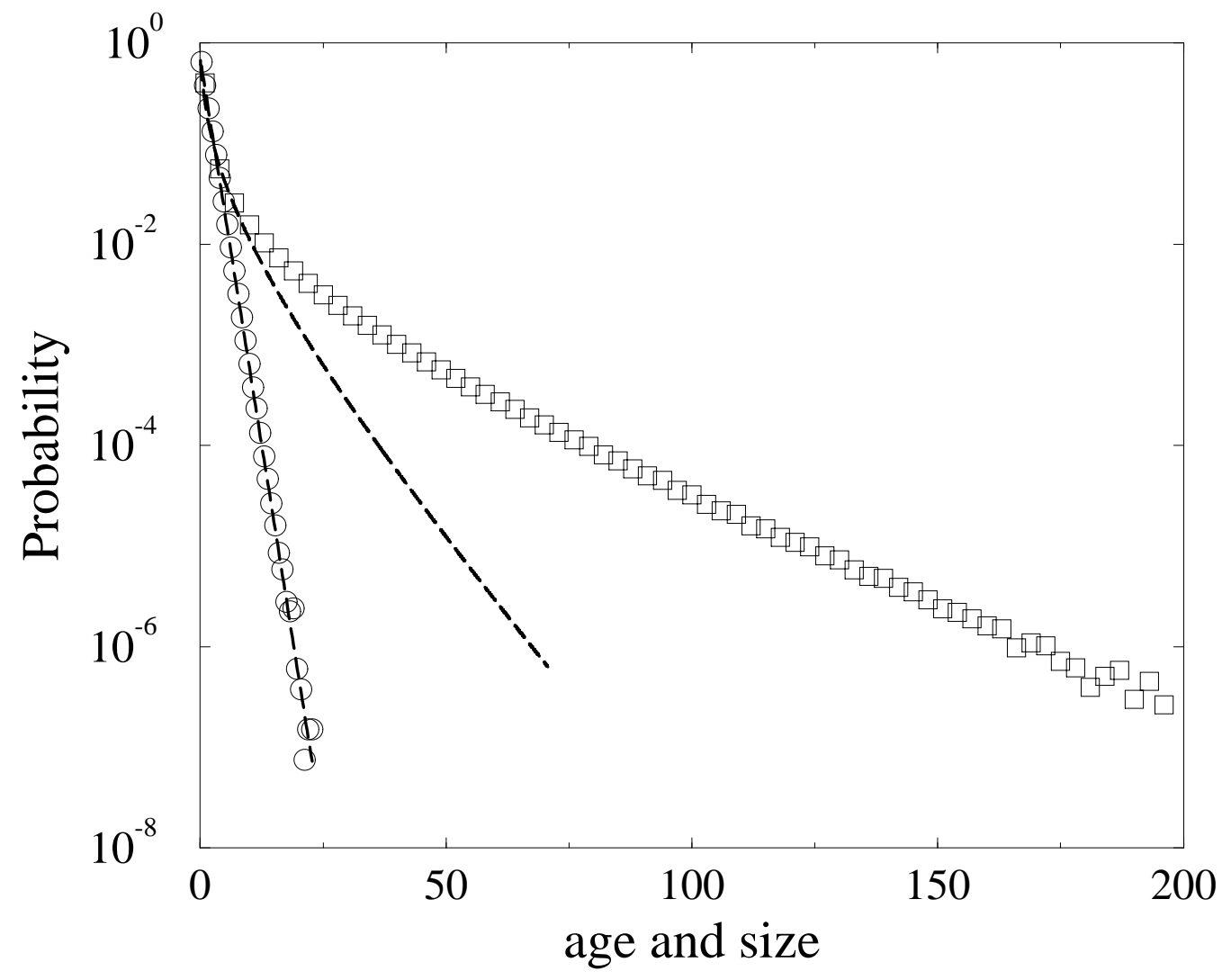

Figure 6. Steady state domain size (o) and age ( $($ ) distributions for the case $\epsilon=1$. The corresponding predictions from the solution to the rate equations are also shown (dashed lines). 


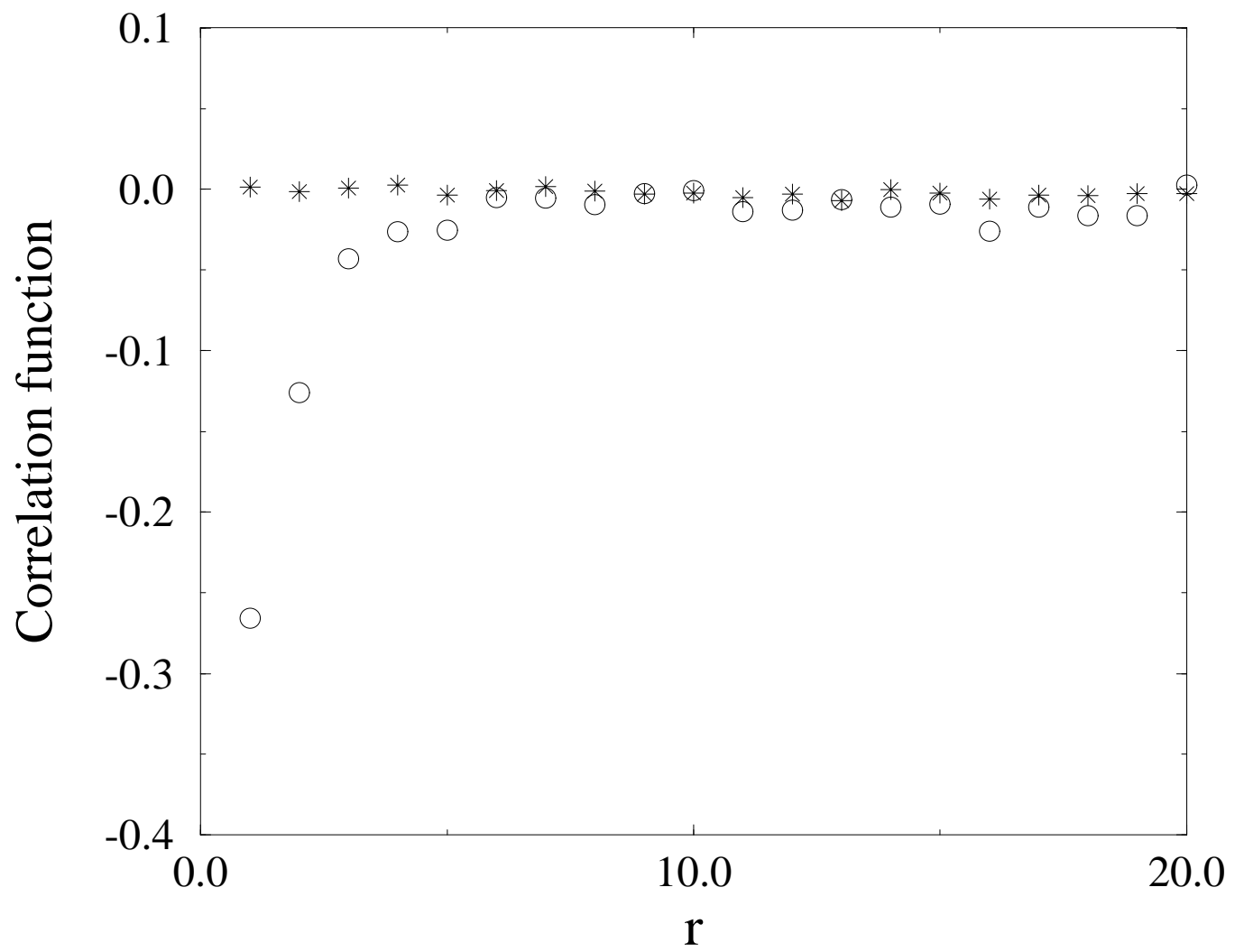

Figure 7. The $r$-dependence of the steady-state size (*) and age (o) correlation functions, $C_{s}(r) \equiv$ $\left\langle s_{i} s_{i+r}\right\rangle /\left\langle s_{i}\right\rangle^{2}-1$ and $C_{\tau}(r) \equiv\left\langle\tau_{i} \tau_{i+r}\right\rangle /\left\langle\tau_{i}\right\rangle^{2}-1$, respectively, for the case of fair war $\epsilon=1$. vfill 


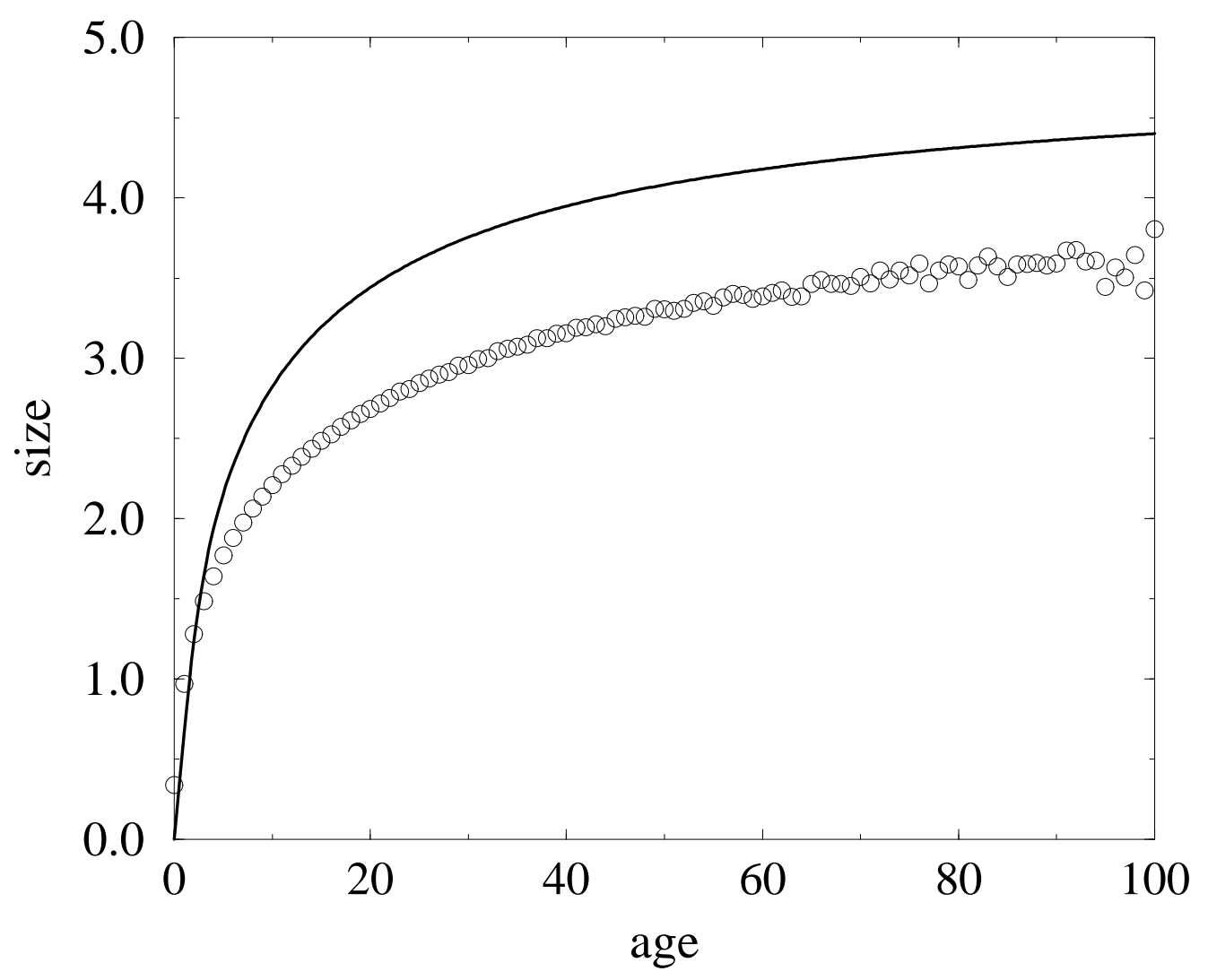

Figure 8. Average domain size as a function of domain age in the steady state for the fair war case of $\epsilon=1$. Shown is are the simulation results (a) and the predictions based on the solution to the rate equations (solid line). 


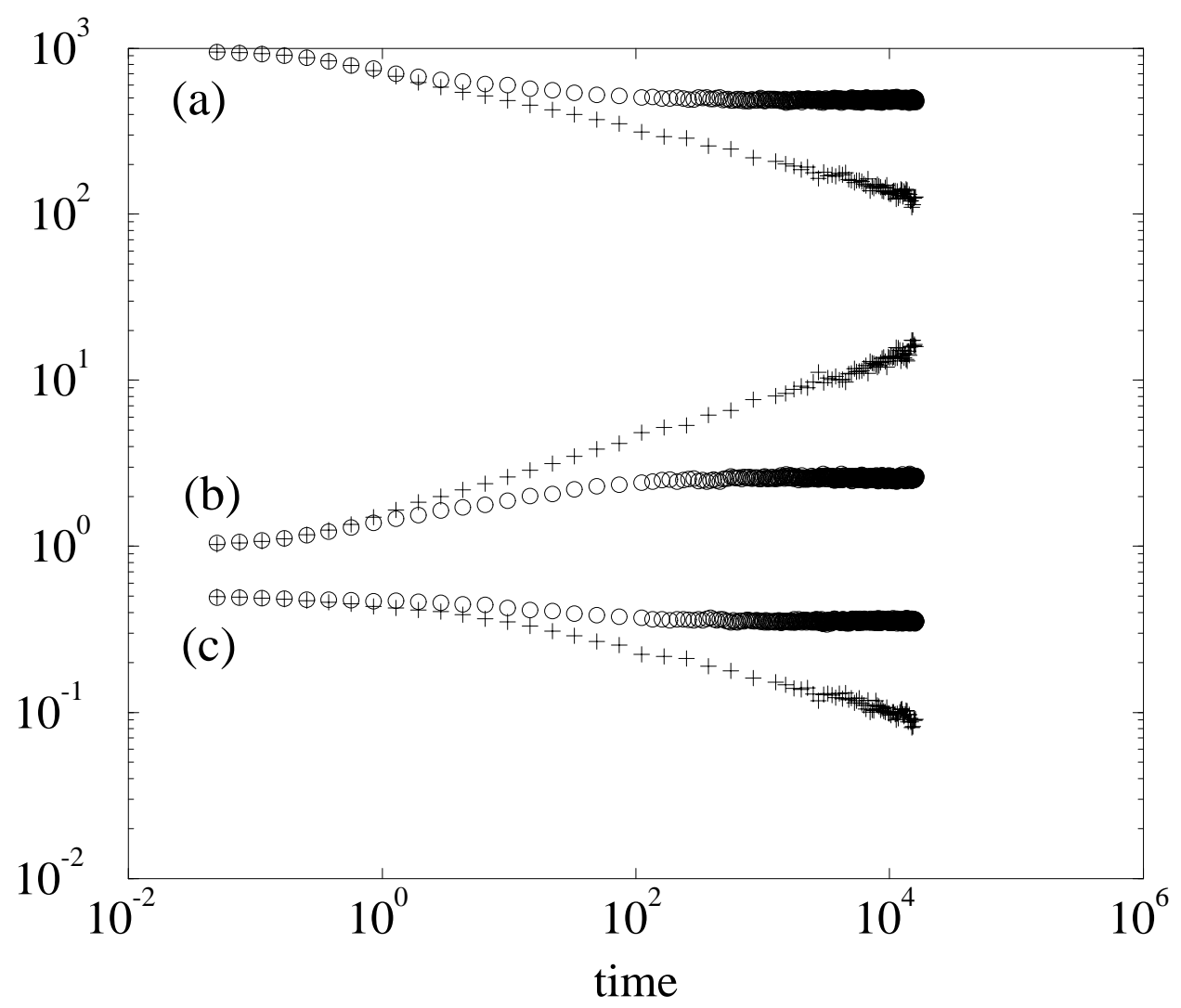

Figure 9. Representative simulation results for time-dependent quantities for a system that has reached a steady state, $\epsilon=2 / 3(\circ)$, and for a system which perpetually coarsens, $\epsilon=2 / 5(+)$. Shown are: (a) the total number of domains, (b) the average domain size, (c) the fraction of empty space, and (d) the average domain age. This data is based on 20 configurations of system with $10^{3}$ domains initially. 


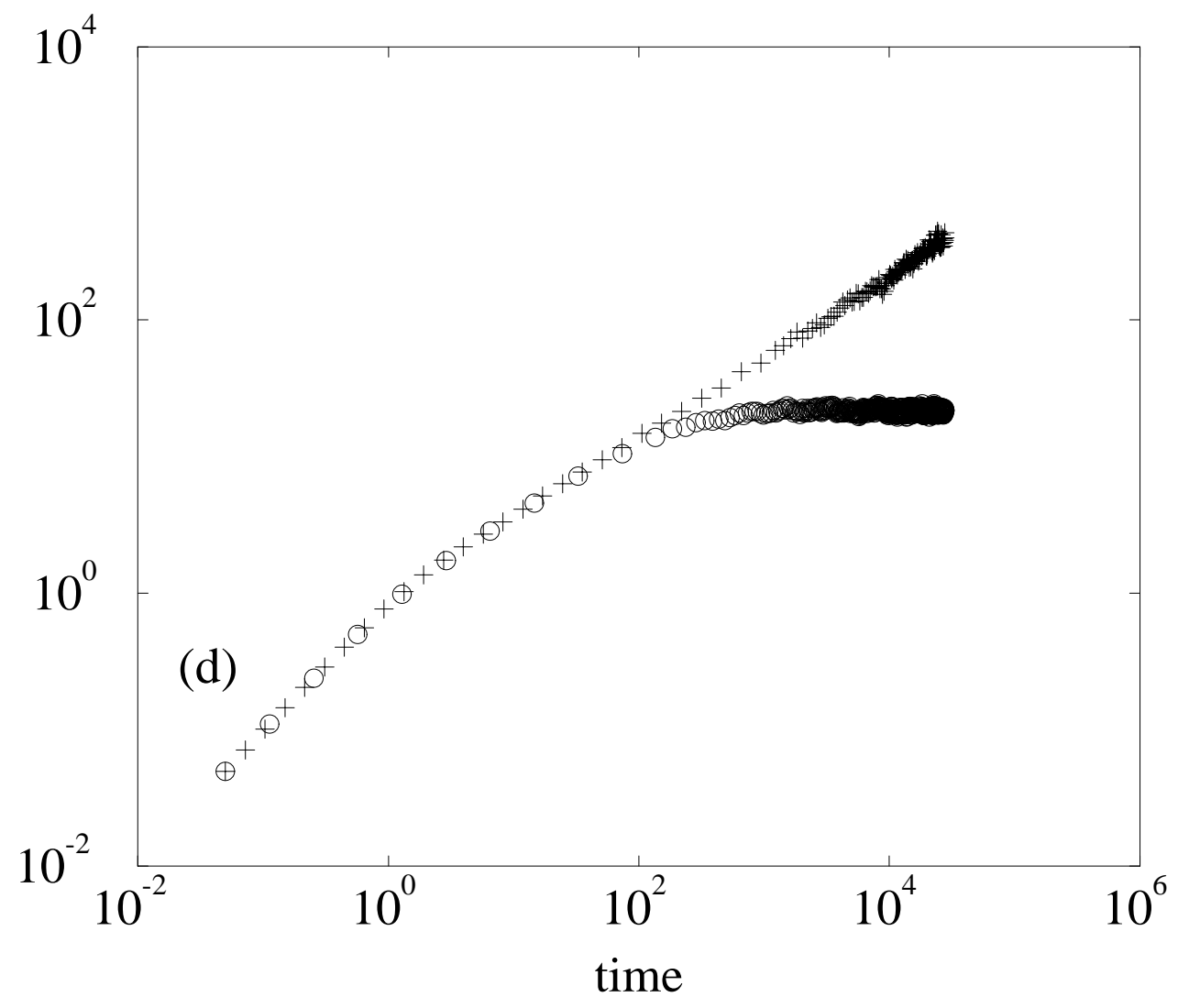

Figure 9 (d) 


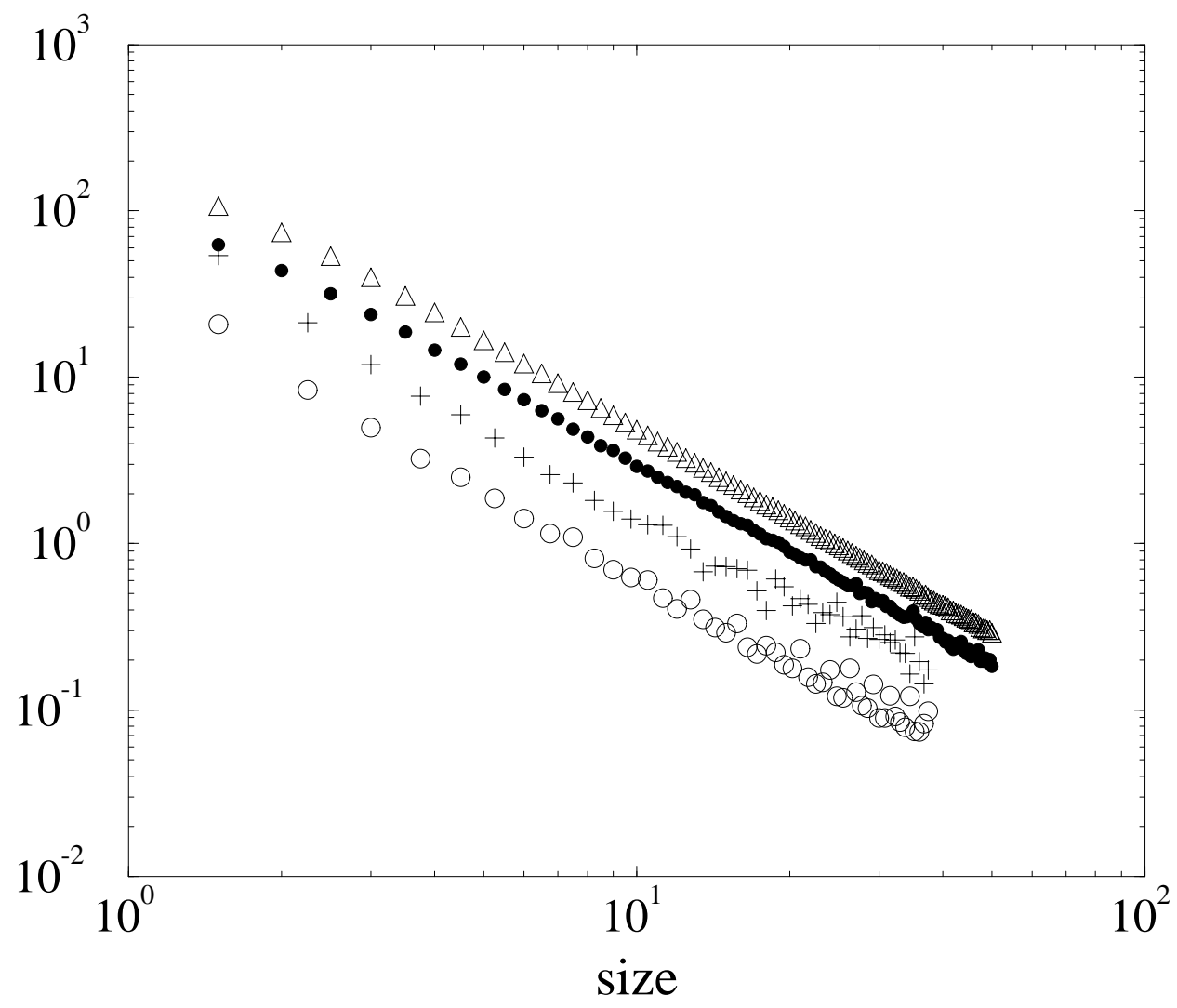

Figure 10. Domain size distribution in the coarsening regime for $\epsilon=0(\circ), \epsilon=0.2(+), \epsilon=0.4$ $(\bullet)$, and $\epsilon=1 / 2 \Delta)$. To distinguish the different data sets, the points for each value of $\epsilon$ have been shifted vertically by a small fixed amount. 


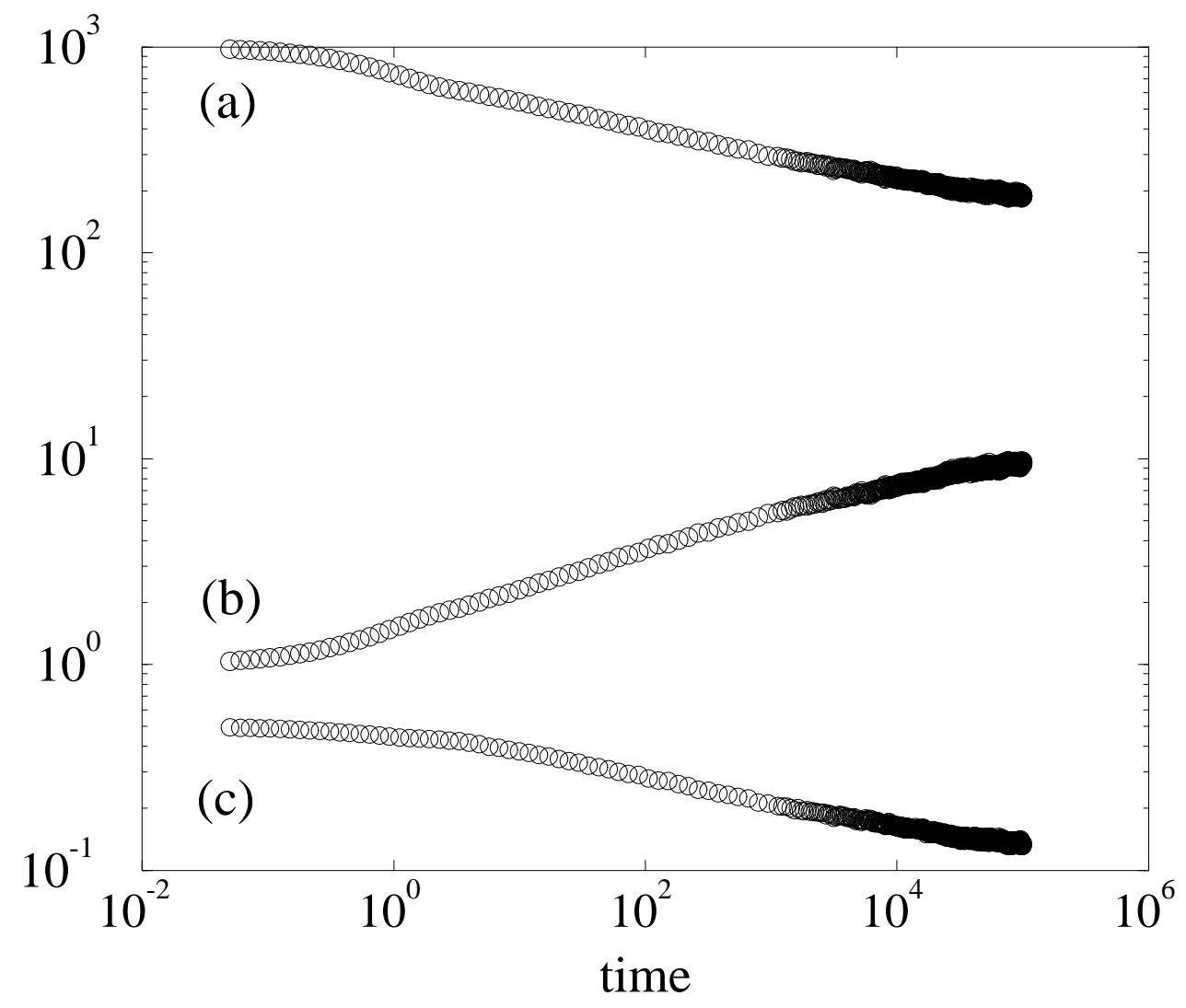

Figure 11. Simulation results for time-dependent quantities for the marginal case of $\epsilon=1 / 2$ Shown are: (a) the total number of domains, (b) the average domain size, (c) the fraction of empty space, and (d) the average domain age. This data is based on 100 configurations of system with $10^{3}$ domains initially. 


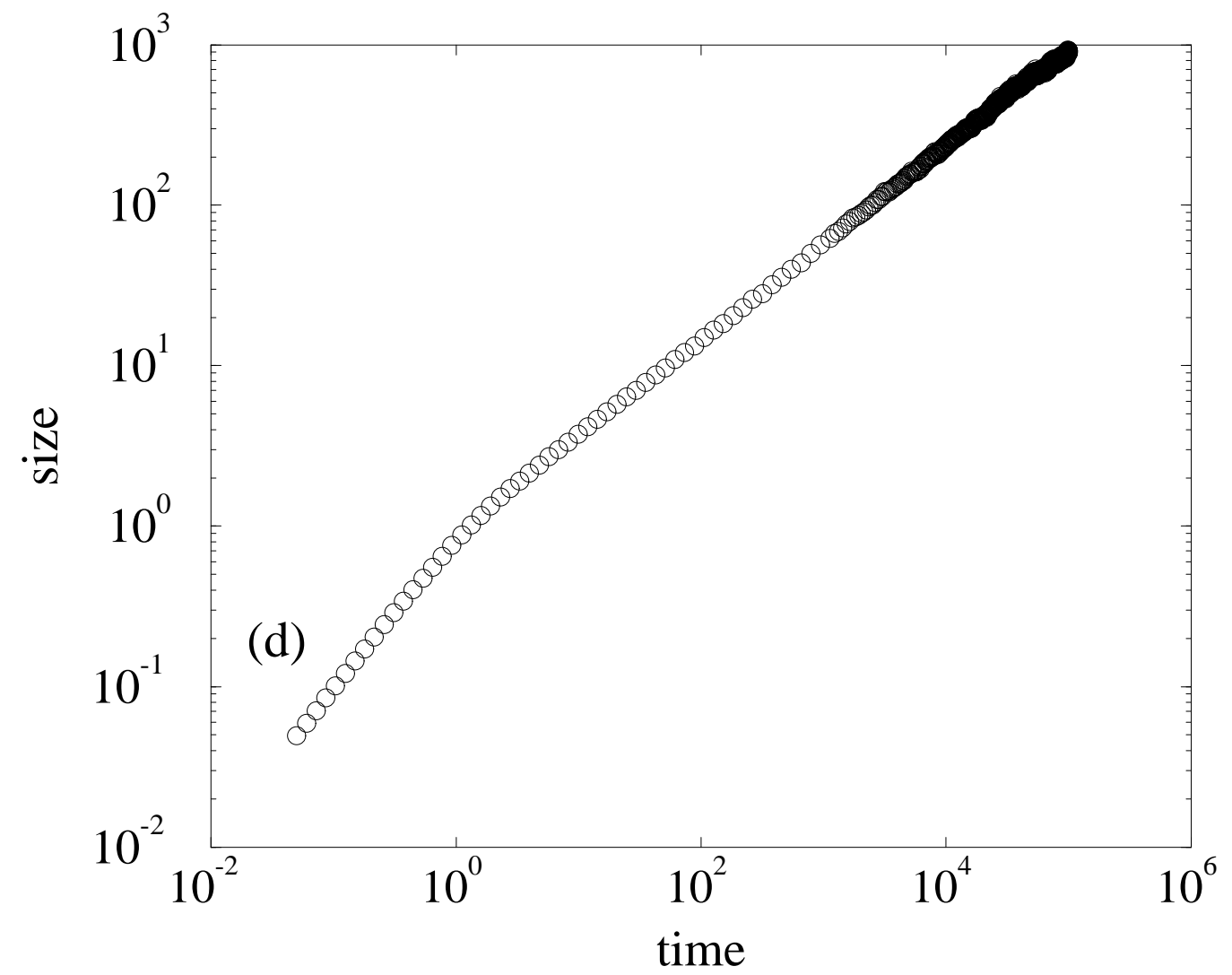

Figure 11 (d) 


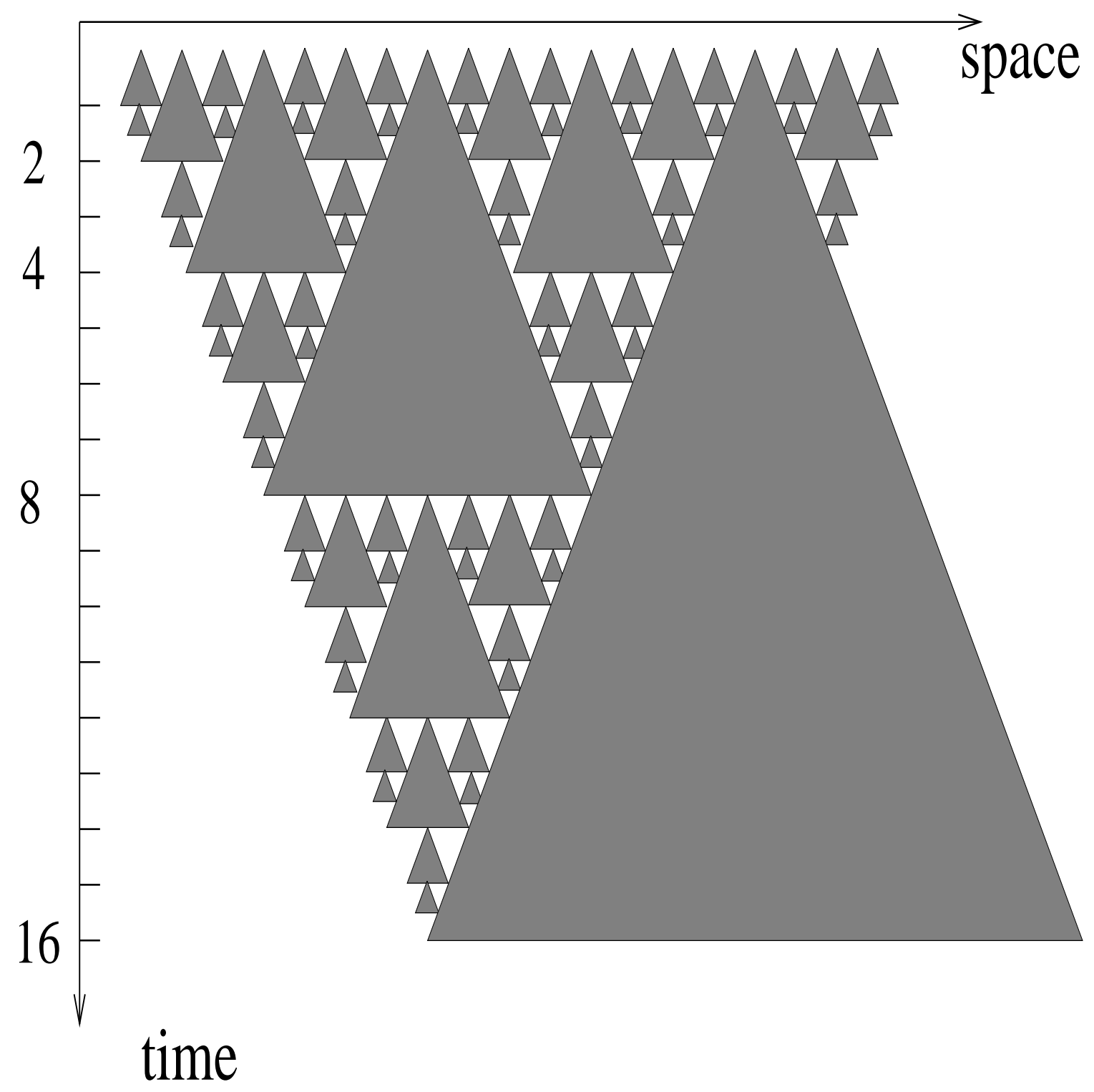

Figure 12. Illustration of the deterministic version of war dynamics for the case $\epsilon=0$. Domains are created at regular lattice points and at regular unit time intervals. When equal size domains meet, a deterministic rule is implement to decide the survivor. 